\title{
Homology of spaces of regular loops in the sphere
}

\author{
DAVID ChataUR \\ JEAN-FRANÇOIS LE BORGNE
}

\begin{abstract}
In this paper we compute the singular homology of the space of immersions of the circle into the $n$-sphere. Equipped with the Chas-Sullivan loop product these homology groups are graded commutative algebras, which we also compute. We enrich Morse spectral sequences for fibrations of free loop spaces together with loop products. This offers some new computational tools for string topology.
\end{abstract}

$55 \mathrm{~N} 45,58 \mathrm{E} 05$

\section{Introduction}

The aim of this paper is to compute the singular homology with integral coefficients of the spaces $\operatorname{Imm}\left(S^{1}, S^{n}\right)$ of immersions of the circle $S^{1}$ into the $n$-sphere $S^{n}$ also called regular loops. These spaces play a key role in knot theory because they detect nontrivial homology classes for spaces of embeddings $\operatorname{Emb}\left(S^{1}, S^{n}\right)$. For example, Cattaneo, Cotta-Ramusino and Longoni [6] use a desingularization map in order to produce nontrivial cohomology classes for spaces of $\operatorname{knots} \operatorname{Emb}\left(S^{1}, \mathbb{R}^{n}\right)$ from cohomology classes of spaces of singular knots, ie spaces of immersions with a fixed number of transverse double points.

Let us explain the strategy of the computation of these homology groups. We consider the space of immersions $\operatorname{Imm}\left(S^{1}, S^{n}\right)$ as an open subset of the free loop space $\mathcal{L} S^{n}=$ $C^{\infty}\left(S^{1}, S^{n}\right)$ and we endow the $n$-sphere with its standard Riemannian metric. We consider the energy functional $E$ defined by

$$
\begin{aligned}
\mathcal{L} S^{n} & \rightarrow \mathbb{R} \\
\gamma & \mapsto \int_{S^{1}}\|\dot{\gamma}(\theta)\|^{2} d \theta
\end{aligned}
$$

and we also consider its restriction $E_{\text {imm }}$ to the space of immersions. From this functional we get a filtration of the space of immersions by the energy level. It follows from Morse theory that this filtration is well understood in the case of the space $\mathcal{L} S^{n}$ of all smooth loops and gives a very efficient way to compute its singular homology. But it is a priori more difficult to handle it for immersions. To this filtration one naturally 
associates a spectral sequence which converges to $H_{*}\left(\operatorname{Imm}\left(S^{1}, S^{n}\right), \mathbb{Z}\right)$. In order to compute the $E_{1}$-term of this spectral sequence it is essential to understand the homology of the pairs $\left(\mathrm{Imm}^{<\lambda_{p}+\epsilon}, \mathrm{Imm}^{<\lambda_{p}-\epsilon}\right)$ where $\lambda_{p}$ is a critical value of $E_{\text {imm }}$ and

$$
\operatorname{Imm}^{<a}:=\left\{\gamma \in \operatorname{Imm}\left(S^{1}, S^{n}\right) / E_{\mathrm{imm}}(\gamma)<a\right\} .
$$

Let $U S^{n}$ be the unit tangent bundle of $S^{n}$ and $D$ be the map

$$
\begin{aligned}
\operatorname{Imm}\left(S^{1}, S^{n}\right) & \rightarrow \mathcal{L} U S^{n} \\
\gamma & \mapsto \frac{\dot{\gamma}}{\|\dot{\gamma}\|} .
\end{aligned}
$$

By a famous result of Hirsch and Smale [18] this map is a weak homotopy equivalence. We have the factorization

$$
E_{\mathrm{imm}}=E \circ \pi \circ D: \operatorname{Imm}\left(S^{1}, S^{n}\right) \stackrel{D}{\longrightarrow} \mathcal{L} U S^{n} \stackrel{\pi}{\longrightarrow} \mathcal{L} S^{n} \stackrel{E}{\longrightarrow} \mathbb{R}
$$

where $\pi=\mathcal{L} p$ is the loop map associated to the canonical projection $p: U S^{n} \rightarrow S^{n}$. Thus our problem reduces to a problem of "fiberwise" Morse theory. We will consider the filtration by the energy level on the space $\mathcal{L} U S^{n}$ induced by the map $E \circ \pi$. From this filtration one gets a Morse-Serre type spectral sequence. To be more precise one has a fibration

$$
\mathcal{L} S^{n-1} \rightarrow \mathcal{L} U S^{n} \rightarrow \mathcal{L} S^{n}
$$

and the filtration on the total space is induced by the Morse filtration (the energy filtration) of the base. We will show that this fiberwise filtration is homotopy equivalent to the filtration induced by $E_{\mathrm{imm}}$.

If we consider this spectral sequence just as a spectral sequence of abelian groups it seems impossible to complete the computation - we need to enrich this spectral sequence with an additional algebraic data. Here string topology enters the game. From the foundational work of Chas and Sullivan [7] one knows that

$$
\mathbb{H}_{*}\left(\mathcal{L} U S^{n}\right):=H_{*+2 n-1}\left(\mathcal{L} U S^{n}\right)
$$

is a graded commutative algebra equipped with the so-called loop product. Hingston and Goresky [10] have proved that this spectral sequence is multiplicative for the loop product.

Moreover we prove that this spectral sequence collapses at the $E_{2}$-term $\left(E_{2}=E_{\infty}\right)$. Thus we are left with some nontrivial extension issues that we solve by comparing the preceding spectral sequences with the Serre spectral sequences associated to the 
fibrations

$$
\begin{aligned}
\Omega U S^{n} & \rightarrow \mathcal{L} U S^{n} \rightarrow U S^{n} \\
\mathcal{L} S^{n-1} & \rightarrow \mathcal{L} U S^{n} \rightarrow \mathcal{L} S^{n} .
\end{aligned}
$$

In fact the Serre spectral sequence associated to the first fibration collapses at the $E_{2}$-term when $n$ is even while the second collapses at the $E_{2}$-term when $n$ is odd; see Le Borgne [14]. But in each case we encounter extension issues that we are able to solve by comparison with the Morse spectral sequence. Thus if we want to complete the computation we need to use these three spectral sequences together. Then we get our main result:

Theorem 0.1 Let $n \geq 2$ be an integer. Then $\mathbb{H}_{*}\left(\operatorname{Imm}\left(S^{1}, S^{2 n}\right)\right) \simeq \mathbb{H}_{*}\left(\mathcal{L} U S^{2 n}\right)$ is isomorphic to the algebra

$$
\mathbb{Z}\left[y_{-2 n}, \alpha_{2 n-2}, \beta_{4 n-2}\right] \otimes \Lambda\left(x_{-4 n+1}, k_{-1}\right) / \mathcal{R}
$$

where $\mathcal{R}$ is the ideal generated by

$$
\left(2 y_{-2 n}, 2 k_{-1}, 2 \alpha_{2 n-2}, x_{-4 n+1} y_{-2 n}, x_{-4 n+1} k_{-1}, y_{-2 n}^{2}, y_{-2 n} k_{-1}-x_{-4 n+1} \alpha_{2 n-2}\right)
$$

and $\mathbb{H}_{*}\left(\operatorname{Imm}\left(S^{1}, S^{2 n+1}\right)\right) \simeq \mathbb{H}_{*}\left(\mathcal{L} U S^{2 n+1}\right)$ is isomorphic to the algebra

$$
\mathbb{Z}\left[v_{2 n}, y_{-2 n}, u_{4 n-2}\right] \otimes \Lambda\left(x_{-2 n-1}, \theta_{-1}\right) /\left(y_{-2 n}^{2}, \theta_{-1} y_{-2 n}, 2 u_{4 n-2} y_{-2 n}\right) .
$$

Remarks Let us give some additional information about the preceding computations.

(1) First let us notice that the evaluation map

$$
\mathrm{ev}_{0}: \operatorname{Imm}\left(S^{1}, S^{n}\right) \rightarrow U S^{n}
$$

has a section geod which associates to each unit tangent vector $u \in U S^{n}$ the unique great circle (the prime geodesic) $\gamma_{u}$ such that $\dot{\gamma}_{u}(0)=u$. In homology $\left(\mathrm{ev}_{0}\right)_{*}$ and (geod) $)_{*}$ are algebra morphisms between the intersection homology algebras $\mathbb{H}_{*}\left(U S^{n}\right)$ and $\mathbb{H}_{*}\left(\operatorname{Imm}\left(S^{1}, S^{n}\right)\right)$. Explicitly one can identify the algebra $\mathbb{H}_{*}\left(U S^{2 n}\right)$ with the subalgebra of $\mathbb{H}_{*}\left(\operatorname{Imm}\left(S^{1}, S^{2 n}\right)\right)$ generated by the classes $y_{-2 n}$ and $x_{-4 n+1}$; in the odd case we can identify $\mathbb{H}_{*}\left(U S^{2 n+1}\right)$ with the subalgebra of $\mathbb{H}_{*}\left(\operatorname{Imm}\left(S^{1}, S^{2 n+1}\right)\right)$ generated by the classes $y_{-2 n}$ and $x_{-2 n-1}$.

(2) Let $\operatorname{Imm}_{u}\left(S^{1}, S^{n}\right)=\operatorname{ev}_{0}^{-1}(u)$ be a fiber of the evaluation map

$$
\mathrm{ev}_{0}: \operatorname{Imm}\left(S^{1}, S^{n}\right) \rightarrow U S^{n}
$$

We consider the inclusion

$$
j: \operatorname{Imm}_{u}\left(S^{1}, S^{n}\right) \rightarrow \operatorname{Imm}\left(S^{1}, S^{n}\right) .
$$


In homology we have the intersection morphism

$$
I=j_{!}: \mathbb{H}_{*}\left(\operatorname{Imm}\left(S^{1}, S^{n}\right)\right) \rightarrow H_{*}\left(\operatorname{Imm}_{u}\left(S^{1}, S^{n}\right)\right)
$$

which corresponds to the classical intersection morphism

$$
\text { Int: } \mathbb{H}_{*}\left(\mathcal{L} U S^{n}\right) \rightarrow H_{*}\left(\Omega U S^{n}\right)
$$

of string topology. In the even case, we have

$$
H_{*}\left(\Omega U S^{2 n}\right) \cong \mathbb{Z}\left[\alpha_{2 n-2}, \beta_{4 n-2}\right] /\left(2 \alpha_{2 n-2}\right)
$$

and the morphism $I$ is given by $I\left(x_{-4 n+1}\right)=I\left(y_{-2 n}\right)=I\left(k_{-1}\right)=0$ and $I\left(\alpha_{2 n-2}\right)=$ $\alpha_{2 n-2}, I\left(\beta_{4 n-2}\right)=\beta_{4 n-2}$. In the odd case we have

$$
H_{*}\left(\Omega U S^{2 n+1}\right) \cong H_{*}\left(\Omega S^{2 n+1}\right) \otimes H_{*}\left(\Omega S^{2 n}\right) \cong \mathbb{Z}\left[v_{2_{n}}\right] \otimes \mathbb{Z}\left[f_{2 n-1}\right]
$$

and $I\left(x_{-2 n-1}\right)=I\left(y_{-2 n}\right)=I\left(\theta_{-1}\right)=0, I\left(v_{2 n}\right)=v_{2 n}, I\left(u_{4 n-2}\right)=\left(f_{2 n-1}\right)^{2}$.

(3) Over the field of rational numbers these computations become easier because rationally $U S^{2 n} \simeq \mathbb{Q} S^{4 n-1}$ and $U S^{2 n+1} \simeq \mathbb{Q} S^{2 n+1} \times S^{2 n}$.

(4) The computation of the loop algebra $\mathbb{H}_{*}\left(\mathcal{L} U S^{n}\right)$ is also related to two other topological problems. One knows that the space $U S^{n}$ is homotopy equivalent to the configuration space $F_{3}\left(S^{n}\right)$ of three points in $S^{n}$. In fact the projection

$$
\operatorname{proj}_{3,1}: F_{3}\left(S^{n}\right) \rightarrow S^{n}
$$

given by $\operatorname{proj}_{3,1}\left(x_{1}, x_{2}, x_{3}\right)=x_{1}$ is fiberwise homotopy equivalent to

$$
p: U S^{n} \rightarrow S^{n}
$$

The computation of the homology of $\mathcal{L} U S^{n}$ is essential in understanding of problems of 3-body type in $S^{n}$; we refer the reader to Fadell and Husseini's monograph [8]. This computation is related to symplectic topology; from recent work of Abbondandolo and Schwarz [1] one knows that the loop algebra $\mathbb{H}_{*}\left(\mathcal{L} U S^{n}\right)$ is isomorphic as an algebra to Floer homology $H F_{*}\left(\left(T U S^{n}\right)^{*}\right)$ of the cotangent bundle of $U S^{n}$ together with the pair of pants product.

Plan of the paper In Section 1, we recall some basic facts about immersion spaces and string topology.

In Section 2, we build our main technical tool the Morse-Serre spectral sequence and show its compatibility with the loop product. We play with various filtrations of loop spaces: the length filtration, the energy filtration and the filtration by the square root of the energy. 
Section 3 is devoted to the computation of the 0 -th column of the Morse-Serre spectral sequence.

In Sections 4 and 5 , we compute $\mathbb{H}_{*}\left(\operatorname{Imm}\left(S^{1}, S^{2 n}\right)\right)$ and $\mathbb{H}_{*}\left(\operatorname{Imm}\left(S^{1}, S^{2 n+1}\right)\right)$.

Acknowledgements The authors thank François Laudenbach for a careful reading of a first version of this paper and the referee for his suggestions and corrections. The authors are supported by the "Laboratoire Paul Painlevé, UMR 8524 de l'Université des Sciences et Technologie de Lille et du CNRS" and by the GDR 2875 "Topologie Algébrique et Applications du CNRS". The first author's research is supported in part by the ANR grant 06-JCJC-0042 "Opérades, Bigèbres et Théories d'Homotopie".

\section{String topology of immersion spaces}

\subsection{Loop product}

We recall the definition of the Chas-Sullivan loop product [7]. Let $M$ be a $d-$ dimensional connected, compact oriented manifold. Moreover we suppose that $M$ is closed that is to say without boundary. Let $\delta_{M}: M \hookrightarrow M \times M, x \mapsto(x, x)$ be the diagonal embedding. We also denote by $\tilde{\delta}_{M}: \mathcal{L} M \times_{M} \mathcal{L} M \hookrightarrow \mathcal{L} M \times \mathcal{L} M$ the embedding of composable loops where

$$
\mathcal{L} M \times_{M} \mathcal{L} M=\left\{\left(\gamma_{1}, \gamma_{2}\right) \in \mathcal{L} M \times \mathcal{L} M / \gamma_{1}(0)=\gamma_{2}(0)\right\} .
$$

We have the pullback diagram

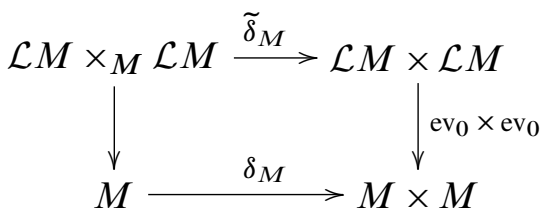

and we have a composition map $\operatorname{comp}_{M}: \mathcal{L} M \times_{M} \mathcal{L} M \rightarrow \mathcal{L} M$ that concatenates the composable loops. To be more precise, the composition map is a continuous map given by $\operatorname{comp}_{M}\left(\gamma, \gamma^{\prime}\right)(t)=\gamma(2 t)$ if $0 \leq t \leq 1 / 2$ and $\operatorname{comp}_{M}\left(\gamma, \gamma^{\prime}\right)(t)=\gamma^{\prime}(2 t-1)$ if $1 / 2 \leq t \leq 1$.

As a pullback of the diagonal embedding, the embedding $\widetilde{\delta_{M}}$ is smooth and finite codimensional, so it is possible to define a shriek map

$$
\widetilde{\delta_{M} !}: H_{*}(\mathcal{L} M \times \mathcal{L} M) \rightarrow H_{*-d}\left(\mathcal{L} M \times_{M} \mathcal{L} M\right) .
$$

Let us briefly recall the construction of this shriek map for a smooth embedding $j: X \hookrightarrow Y$ of finite codimension $k$, where $X$ and $Y$ can be, and this is our case, 
infinite dimensional manifolds. We suppose that this embedding is co-oriented, that is to say the normal bundle of $j$ is an oriented vector bundle. For such an embedding we suppose that there is a tubular neighbourhood $\operatorname{Tub}(X)$ of $j(X)$ that is diffeomorphic to the total space of the disc bundle $D_{k}(X) \rightarrow X$ of the normal bundle of $j$ and let $S_{k-1}(X) \rightarrow X$ be its associated sphere bundle. Then $j_{!}: H_{*}(Y) \rightarrow H_{*-k}(X)$ is defined as the composition of

(a) the inclusion of pairs $H_{*}(Y) \rightarrow H_{*}(Y, Y-j(X))$,

(b) the excision isomorphism

$$
H_{*}(Y, Y-j(X)) \rightarrow H_{*}(\operatorname{Tub}(X), \operatorname{Tub}(X)-j(X)),
$$

(c) an isomorphism induced by an homotopy equivalence

$$
H_{*}(\operatorname{Tub}(X), \operatorname{Tub}(X)-j(X)) \rightarrow H_{*}\left(D_{k}(X), S_{k-1}(X)\right),
$$

(d) the Thom isomorphism $H_{*}\left(D_{k}(X), S_{k-1}(X)\right) \rightarrow H_{*-k}(X)$ that is the composition of the cap product with the Thom class $\tau \in H_{k}\left(D_{k}(X), S_{k-1}(X)\right)$ with the map $\pi_{*}$ induced by the canonical projection $\pi: D_{k}(X) \rightarrow X$. If the embedding is not co-oriented we have to use singular homology with local coefficients.

The Chas-Sullivan loop product $\mu$ is defined as the composition map

$\mu: H_{*}(\mathcal{L} M) \otimes H_{*}(\mathcal{L} M) \stackrel{\times}{\rightarrow} H_{*}(\mathcal{L} M \times \mathcal{L} M)$

$$
\stackrel{\widetilde{\delta_{M}}}{\longrightarrow} H_{*-d}\left(\mathcal{L} M \times_{M} \mathcal{L} M\right) \stackrel{\mathrm{comp}_{M *}}{\longrightarrow} H_{*-d}(\mathcal{L} M) .
$$

where $\times$ denotes the cross product. For other constructions of this product we refer the reader to Sullivan's survey paper [19]. Let us recall some basic facts about this product:

(1) When suitably regraded ie we define $\mathbb{H}_{*}(\mathcal{L} M):=H_{*+d}(\mathcal{L} M)$ the loop product is unitary and commutative.

(2) The loop product is compatible with the intersection product of $M$. Let us recall that $\mathbb{H}_{*}(M):=H_{*+d}(M)$ together with the intersection product is a graded commutative algebra called the intersection algebra. The evaluation map ev $\mathrm{v}_{0}: \mathcal{L} M \rightarrow M$ and its section $c: M \rightarrow \mathcal{L} M$ the constant loop map induce two morphisms of algebras

$$
\left(\mathrm{ev}_{0}\right)_{*}: \mathbb{H}_{*}(\mathcal{L} M) \rightleftarrows \mathbb{H}_{*}(M):(c)_{*} .
$$

The algebra $\mathbb{H}_{*}(M)$ is isomorphic to a subalgebra of the loop algebra, the unit of $\mu$ is equal to $(c)_{*}([M])$ where $[M]$ is the fundamental class of $M$. 
(3) The composition of based loops induces in homology

$$
\sharp: H_{*}(\Omega M) \otimes H_{*}(\Omega M) \rightarrow H_{*}(\Omega M)
$$

the Pontryagin product which has a unit and is associative. The inclusion $j: \Omega M \rightarrow \mathcal{L} M$ can be considered as a codimension $d$ embedding. Thus one can define a shriek map

$$
\text { Int }:=j_{!}: \mathbb{H}_{*}(\mathcal{L} M) \rightarrow H_{*}(\Omega M) .
$$

This is a morphism of algebras called the intersection morphism.

(4) There are several constructions of the loop product, some are purely algebraic, others use stable homotopy theory. A nice description of the loop product has been given by F Laudenbach [13], who uses his approach to give a direct construction of the multiplicative structures considered by Hingston and Goresky [10] and in this paper.

\subsection{Hirsch and Smale's theorem and loop products}

Let $M$ be a smooth Riemannian manifold and let $U M$ be the unit tangent bundle. We consider the evaluation map

$$
\mathrm{ev}_{0}: \operatorname{Imm}\left(S^{1}, M\right) \rightarrow U M
$$

defined by $\mathrm{ev}_{0}(\gamma)=\dot{\gamma}(0) /\|\dot{\gamma}(0)\|$. This evaluation map is a fibration, this was first proved by S Smale [17, Theorem B]. But one can prove a stronger result, in fact this map is locally trivial. We use a theorem of R S Palais [15, Theorem A]: the group of diffeomorphisms $\operatorname{Diff}(M)$ acts on $\operatorname{Imm}\left(S^{1}, M\right)$ and $U M$, the evaluation map is equivariant and $U M$ is a $\operatorname{Diff}(M)$-space admitting local cross-sections (let $x \in U M$ be a unitary tangent vector there is a map $\chi$ of a neighborhood $\mathrm{U}$ of $x$ into $\operatorname{Diff}(M)$ such that $\chi(u)(x)=u$ for all $u \in U)$, then by [15, Theorem A] the map ev ${ }_{0}$ is locally trivial.

We have the pullback diagram

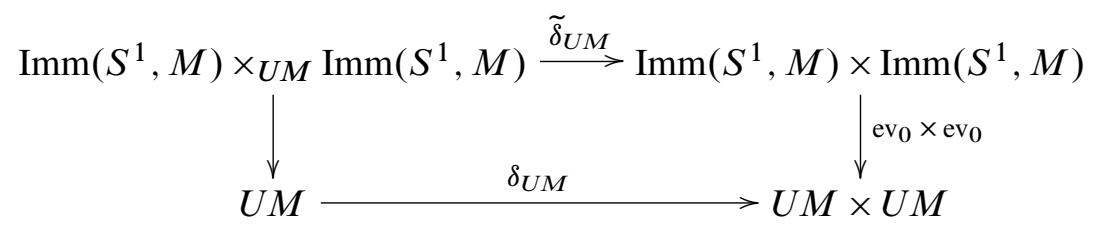

and a composition map

$$
\text { comp: } \operatorname{Imm}\left(S^{1}, M\right) \times_{U M} \operatorname{Imm}\left(S^{1}, M\right) \rightarrow \operatorname{Imm}\left(S^{1}, M\right) .
$$


One can proceed as in the preceding section and define an immersion product and an immersion algebra

$$
\mathbb{H}_{*}\left(\operatorname{Imm}\left(S^{1}, M\right)\right):=H_{*+2 d-1}\left(\operatorname{Imm}\left(S^{1}, M\right)\right) .
$$

We also notice that if $u \in U M$ and if $\operatorname{Imm}_{u}\left(S^{1}, M\right):=\left(\operatorname{ev}_{0}\right)^{-1}(u)$ we have an intersection morphism

$$
\mathbb{H}_{*}\left(\operatorname{Imm}\left(S^{1}, M\right)\right) \rightarrow H_{*}\left(\operatorname{Imm}_{u}\left(S^{1}, M\right)\right) .
$$

Remark The definition of the map comp needs to be modified slightly. First we notice that the concatenation of two composable immersions is not well defined. Let $\gamma$ and $\gamma^{\prime}$ be two composable immersions. At $t=0$ the loops $\gamma$ and $\gamma^{\prime}$ do not have the same tangent vector but their normalizations are the same by definition of the space of composable immersions. Thus after concatenation of the composable immersions we reparametrize the loop by its arc length. We also notice that the concatenation is a piecewise $C^{2}$-path. This space of piecewise $C^{2}$-immersions is homotopy equivalent to space $\operatorname{Imm}\left(S^{1}, M\right)$ of $C^{\infty}$-immersions. We should also work with piecewise $C^{2}$-paths rather than $C^{\infty}$-paths (all these spaces of loops and paths are homotopy equivalent - see Palais [16]).

Now we relate the immersion algebra to the string topology intersection algebra of $U M$. By Hirsch and Smale's homotopy theory of immersions $[11 ; 18]$ we know that the differential

$$
D: \operatorname{Imm}\left(S^{1}, M\right) \rightarrow \mathcal{L} U M
$$

is a homotopy equivalence. We have a map of fibrations

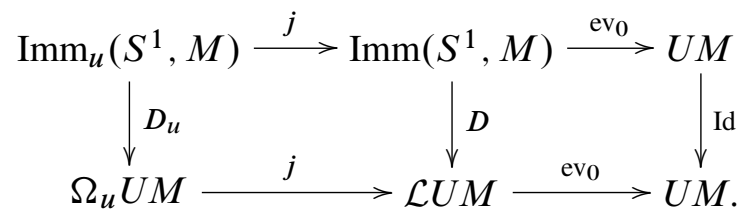

This map is a morphism of fiberwise monoids in the sense of Gruher and Salvatore [9]. Thus we have:

Lemma 1.1 Let $M$ be a connected closed smooth manifold then $D_{*}$ gives an isomorphism of algebras between the immersion algebra $\mathbb{H}_{*}\left(\operatorname{Imm}\left(S^{1}, M\right)\right)$ and the loop algebra $\mathbb{H}_{*}(\mathcal{L} U M)$.

Let us suppose that $M$ is 1 -connected. Following Cohen-Jones-Yan, the Serre spectral sequence associated to $\mathrm{ev}_{0}$ is multiplicative with respect to the loop product. 
This structure involves the intersection product on the homology of the base and the Pontryagin product on the homology of the fiber. Our first computational tool is the multiplicative spectral sequence

$$
\mathbb{E}_{p, q}^{2}:=\mathbb{H}_{p}\left(U M, H_{q}(\Omega U M)\right) \Rightarrow \mathbb{H}_{p+q}(\mathcal{L} U M) .
$$

Let us consider the unit tangent bundle

$$
S^{d-1} \rightarrow U M \stackrel{p}{\longrightarrow} M
$$

Our second computational tool is associated to the fibration $\pi:=\mathcal{L} p$

$$
\mathcal{L} S^{d-1} \rightarrow \mathcal{L} U M \stackrel{\pi}{\longrightarrow} \mathcal{L} M .
$$

The second's author has proved in his $\mathrm{PhD}$ thesis [14] that the Serre spectral sequence of this fibration is multiplicative for the loop product. We have

$$
\mathbb{E}_{p, q}^{2}:=\mathbb{H}_{p}\left(\mathcal{L} M, \mathbb{H}_{q}\left(\mathcal{L} S^{d-1}\right)\right) \Rightarrow \mathbb{H}_{p+q}(\mathcal{L} U M) .
$$

If $U_{m} \subset M$ is a neighbourhood of a point $m \in M$ diffeomorphic to $\mathbb{R}^{d}$, the differential maps the space $\operatorname{imm}\left(S^{1}, U_{m}\right)$ into $\mathcal{L} U \mathbb{R}^{d}$. Denote by triv the trivialization map

$$
\text { triv: } U \mathbb{R}^{d} \rightarrow \mathbb{R}^{d} \times S^{d-1}
$$

and define tr: $\mathcal{L} U \mathbb{R}^{d} \rightarrow \mathcal{L} S^{d-1}$ as $\operatorname{tr}:=\mathcal{L}\left(\mathrm{pr}_{2}\right.$ otriv). If $f: \operatorname{Imm}\left(S^{1}, M\right) \rightarrow \mathcal{L} U M$ is the canonical inclusion, then we have a diagram

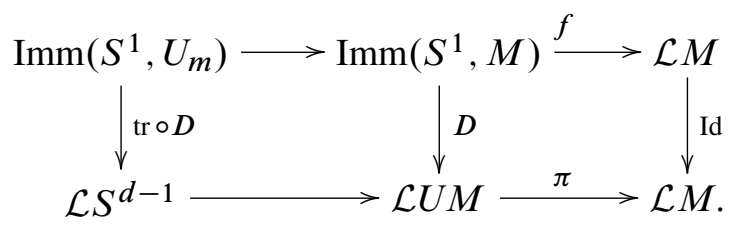

In this diagram each vertical map is a weak homotopy equivalence. The right hand side square is strictly commutative whereas the left hand side square is commutative only up to homotopy.

\subsection{Loop algebra for spheres}

We have seen that the loop algebra of spheres plays a major role in the determination of the immersion algebra of a manifold. Let us recall Cohen, Jones and Yan's computation of these algebras:

$$
\mathbb{H}_{*}\left(\mathcal{L} S^{n}\right) \cong\left\{\begin{array}{cl}
\Lambda(a) \otimes \mathbb{Z}[u] & \text { for } n \text { odd } \\
(\Lambda(b) \otimes \mathbb{Z}[a, v]) /\left(a^{2}, a b, 2 a v\right) & \text { for } n \text { even }
\end{array}\right.
$$


In the Serre spectral sequence of

$$
\Omega S^{n} \rightarrow \mathcal{L} S^{n} \rightarrow S^{n}
$$

we have $a \in \mathbb{H}_{-n}\left(\mathcal{L} S^{n}\right) \cong \mathbb{E}_{-n, 0}^{\infty}, b \in \mathbb{H}_{-1}\left(\mathcal{L} S^{n}\right) \cong \mathbb{E}_{-n, n-1}^{\infty}, u \in \mathbb{H}_{n-1}\left(\mathcal{L} S^{n}\right) \cong$ $\mathbb{E}_{0, n-1}^{\infty}$ and $v \in \mathbb{H}_{2 n-2}\left(\mathcal{L} S^{n}\right) \cong \mathbb{E}_{0,2 n-2}^{\infty}$. In the next section we will show how to recover these results using Morse theory.

\section{Loop product and Morse theory}

\subsection{Morse theory for loop spaces}

In this section we recall some basic facts about Morse-Bott theory for loop spaces, we refer the reader to Bott's papers $[3 ; 5]$. Let $W$ be a manifold and $f$ be a real valued function on $W$. A connected submanifold $N \subset W$ is said to be a nondegenerate critical manifold of $W$ if the following conditions are satisfied.

(1) Each point $p \in N$ is a critical point of $f$.

(2) The Hessian of $f$ is nondegenerate in the normal direction to $N$.

Spelled out this last condition takes this form:

(3) We consider a small tubular $\epsilon$-neighborhood $W_{\epsilon}(N)$ of $N$, which is fibered over $N$ by the normal discs swept out by geodesics of length $\leq \epsilon$ in the normal direction to $N$, relative to some Riemannian structure on $W$. Then, condition (2) is equivalent to the following assumption:

$f$ restricted to each normal disc is nondegenerate.

In this case we decompose the normal bundle $v N$ into a positive and a negative part

$$
v N=v^{+} N \oplus v^{-} N,
$$

where $v_{p}^{+} N$ and $v_{p}^{-} N$ are respectively spanned by the positive and negative eigendirections of the Hessian of $f$. The fiber dimension of $v^{-} N$ will be denoted $\alpha^{N}$ and referred to as the index of $N$ rel $f$. We refer to this conditions as "the Bott nondegeneracy conditions" and say that $f$ is Morse-Bott.

Let us concentrate on the case of the free loop space $\mathcal{L} M$ of a $d$-dimensional compact Riemannian manifold $M$. We work with piecewise smooth loops, we consider the 
Sobolev class $H^{1}\left(S^{1}, M\right)$. Such a model has the advantage to be a Hilbert manifold. The function we consider is the energy functional

$$
E: \mathcal{L} M \rightarrow \mathbb{R}, \gamma \mapsto \int_{S^{1}}\|\dot{\gamma}(t)\|^{2} d t
$$

The critical points of the energy are the closed geodesics. In order to use Morse theory we pick a metric on $M$ such that this function is Morse-Bott. Thus the critical points of $E$ are collected on compact critical manifolds that satisfy the Bott nondegeneracy conditions.

In general this condition holds for a generic metric on $M$. According to R Bott [5] this genericity follows already from general position arguments in Morse's work. W Ziller [20, Theorem 2] proves that globally symmetric spaces satisfy this condition. This result was first proved by $\mathrm{R}$ Bott for the loop space with fixed endpoints $\Omega(M, p, q)$ of a globally symmetric space.

Let $0=\lambda_{0}<\lambda_{1}<\cdots$ be the critical values of the energy function $E$ and

$$
\left.\left.\mathcal{L} M^{\leq \lambda_{i}}:=E^{-1}(]-\infty ; \lambda_{i}\right]\right) \text {. }
$$

The spaces $\mathcal{L} M^{\leq \lambda_{i}}$ give rise to a filtration of $\mathcal{L} M$ that provides a filtration of the singular chain complex $C_{*}(\mathcal{L} M)$. We define

$$
F_{p} C_{p+q}(\mathcal{L} M):=C_{p+q}\left(\mathcal{L} M^{\leq \lambda_{p}}\right) .
$$

Now let us identify the graded module associated to this filtration. Let $\Sigma_{r}$ be the critical submanifold associated to the critical value $\lambda_{r}$ namely

$$
\Sigma_{r}=\left\{\gamma \in \mathcal{L} M / E(\gamma)=\lambda_{r} \text { and } d E(\gamma)=0\right\} .
$$

The manifold $\Sigma_{r}$ is finite dimensional.

For the moment we assume that the critical submanifold is connected. The tangent bundle of $\mathcal{L} M$ restricted to $\Sigma_{r}$ splits into three parts

$$
\left.T \mathcal{L} M\right|_{\Sigma_{r}} \simeq \mu_{r}^{-} \oplus \mu_{r}^{0} \oplus \mu_{r}^{+}
$$

corresponding to the signature of the hessian of $E$ at the point $\gamma$. The main result of Morse theory for free loop spaces is that there is a homotopy equivalence

$$
\mathcal{L} M^{\leq \lambda_{r}} \simeq \mathcal{L} M^{\leq \lambda_{r-1}} \cup_{f_{r}} D\left(\mu_{r}^{-}\right)
$$

for a gluing map $f_{r}: S\left(\mu_{r}^{-}\right) \rightarrow \mathcal{L} M^{\leq \lambda_{r-1}}$. The quotient $\mathcal{L} M^{\leq \lambda_{r}} / \mathcal{L} M^{\leq \lambda_{r-1}}$ is homotopically equivalent to the Thom space $\operatorname{Th}\left(\mu_{r}^{-}\right)$of the bundle $\mu_{r}^{-}$. This proves that

$$
F_{p} C_{p+*}(\mathcal{L} M) / F_{p-1} C_{p+*}(\mathcal{L} M)=C_{p+*}\left(\mathcal{L} M^{\leq \lambda_{p}}, \mathcal{L} M^{\leq \lambda_{p-1}}\right)
$$


is quasi-isomorphic to $\widetilde{C}_{p+*}\left(\operatorname{Th}\left(\mu_{p}^{-}\right)\right)$. The case of several isolated critical manifolds can be treated in the same fashion.

The filtration leads to a homology spectral sequence called the Morse spectral sequence.

Theorem 2.1 Let $M$ be a metric such that the energy is a Morse-Bott function. The energy filtration of $C_{*}(\mathcal{L} M)$ induces a spectral sequence

$$
\left\{E_{*, *}^{r}(\mathcal{M})(\mathcal{L} M)\right\}_{r \in \mathbb{N}}
$$

converging to $H_{*}(\mathcal{L} M)$ :

$$
E_{p, q}^{r}(\mathcal{M})(\mathcal{L} M) \Rightarrow H_{p+q}(\mathcal{L} M) .
$$

We suppose that the critical set $\Sigma_{p}$ with critical value $\lambda_{p}$ is a union of connected nondegenerate manifolds $N_{i}$ and that $\mu_{i}^{-}$denotes the negative part of $\left.T \mathcal{L} M\right|_{N_{i}}$. Then the $E^{1}$-page $E_{p, q}^{1}(\mathcal{M})(\mathcal{L} M)=H_{p+q}\left(\mathcal{L} M^{\leq \lambda_{p}}, \mathcal{L} M^{\leq \lambda_{p-1}}\right)$ is isomorphic to the reduced homology $\bigoplus_{i} \widetilde{H}_{p+q}\left(\operatorname{Th}\left(\mu_{i}^{-}\right)\right)$.

Remark Let $N_{i}$ be a connected component of a critical set $\Sigma_{p}$, the theorem above and the Thom isomorphism gives an isomorphism

$$
H_{*}\left(\mathcal{L} M^{\leq \lambda_{p}}, \mathcal{L} M^{\leq \lambda_{p-1}}\right) \cong \widetilde{H}_{*-a_{p}}\left(N_{i}, \mathbb{Z}\right)
$$

only when the negative bundle $\mu_{i}^{-}$of dimension $a_{p}$ is oriented. Otherwise one has to use coefficients in the orientation bundle of $\mu_{i}^{-}$.

\subsection{Loop products in the Morse spectral sequence}

In this section we lift the Chas-Sullivan product at the chain level in order to get a multiplicative Morse spectral sequence. F Laudenbach [13] has given an alternative construction of the multiplicative structure of this spectral sequence.

The use of the length filtration The composition map comp $M$ is not compatible with the energy filtration. Hingston and Goresky [10] changed this map by a parameterized composition map. However, if one wants to use the map comp ${ }_{M}$ one has to work with the length filtration rather than the energy filtration. Because of its geometric flavour this filtration is more natural and will be very useful in the fiberwise case.

Let $\mathcal{L} M_{l}^{\leq a}$ be the space of loops $\gamma$ of length $L(\gamma) \leq a$. By the Cauchy-Schwarz inequality one has the inclusion

$$
\mathcal{L} M^{\leq a} \rightarrow \mathcal{L} M_{l}^{\leq a}
$$


This inclusion is a homotopy equivalence and this map has a homotopy inverse. Let us be more precise we consider the subspace $\mathcal{A}(M)^{\leq a} \subset \mathcal{L} M$ of loops $\gamma$ parametrized proportionally to arc length such that $L(\gamma) \leq a$. If $\gamma \in \mathcal{A}(M) \leq a$ the length $L(\gamma)$ is equal to $F(\gamma)$. One can also filter the space $\mathcal{A}(M)$ by the length of loops by [10, Proposition 2.2] this filtration is homotopy equivalent to the Morse filtration of $\mathcal{L} M$ by $F$ and to the length filtration of $\mathcal{L} M$. In fact the inclusion $\mathcal{A}(M) \subset \mathcal{L} M$ has a homotopy inverse

$$
A: \mathcal{L} M \rightarrow \mathcal{A}(M)
$$

which associates to any path the same path parametrized proportionally to arclength, with the same basepoint. This map does not change the length of the loop. We have the homotopy equivalences of pairs

$$
\left(\mathcal{L} M^{\leq a}, \mathcal{L} M^{\leq b}\right) \leftarrow\left(\mathcal{A}(M)^{\leq a}, \mathcal{A}(M)^{\leq b}\right) \rightarrow\left(\mathcal{L} M_{l}^{\leq a}, \mathcal{L} M_{l}^{\leq b}\right) .
$$

From now on, we use the length filtration in order to take advantage of the formula

$$
L\left(\operatorname{comp}_{M}\left(\gamma, \gamma^{\prime}\right)\right)=L(\gamma)+L\left(\gamma^{\prime}\right) .
$$

By abuse of notation we use the same notation for the length filtration and the energy filtration.

The chain level product Let $j: X \rightarrow Y$ be a co-oriented finite codimensional embedding and let $C_{*}(X)$ be the singular chain complex of $X$. If we chose $\hat{\tau} \in$ $C^{k}\left(D_{k}(X), S_{k-1}(X)\right)$ representing the Thom class at the chain level, we can define $\hat{j} !: C_{*}(Y) \rightarrow C_{*-k}(X)$ that induces $j$ ! in homology whatever the choice of the cocycle representing $\tau$ is. So let us consider the Chas-Sullivan loop product at the chain level; we have the following diagram:

$$
\begin{aligned}
& C_{p+q}\left(\mathcal{L} M^{\leq \lambda_{p}}\right) \otimes C_{p^{\prime}+q^{\prime}}\left(\mathcal{L} M^{\leq \lambda_{p^{\prime}}}\right) \\
& \downarrow \times \\
& C_{p+q+p^{\prime}+q^{\prime}}\left(\mathcal{L} M^{\leq \lambda_{p}} \times \mathcal{L} M^{\leq \lambda_{p^{\prime}}}\right) \\
& \underset{\delta_{M}}{ } \\
& C_{p+q+p^{\prime}+q^{\prime}-d}\left(\mathcal{L} M^{\leq \lambda_{p}} \times_{M} \mathcal{L} M^{\leq \lambda_{p^{\prime}}}\right) \\
&\left(\operatorname{comp}_{M}\right)_{*} \\
& C_{p+q+p^{\prime}+q^{\prime}-d}\left(\mathcal{L} M^{\leq} \lambda_{p}+\lambda_{p^{\prime}}\right)
\end{aligned}
$$


Condition (Cl) Let $M$ be as in the preceding section a $d$-dimensional compact oriented Riemannian manifold. The main hypothesis that we need here is that all geodesics $\gamma$ are closed and simply periodic with the same prime length $l$. We recall that this means that $\gamma(0)=\gamma(1), \dot{\gamma}(0)=\dot{\gamma}(1), \gamma$ is injective on $(0,1)$ and the length of $\gamma$ namely $L(\gamma)$ is equal to $l$ if $\gamma$ is prime.

Moreover we suppose that all the negative bundles of critical sets are oriented. We call this Condition $(\mathrm{Cl})$. Spheres, complex projective spaces, more generally 1-connected globally symmetric spaces of rank one all satisfy this condition. In fact $\mathrm{R}$ Bott proved in [4] that the singular cohomology of a space that satisfies Condition $(\mathrm{Cl})$ is isomorphic to the cohomology of an irreducible symmetric space of rank 1 .

The fundamental point for us is that Condition $(\mathrm{Cl})$ implies that $\lambda_{r}+\lambda_{r^{\prime}}=r l+r^{\prime} l=$ $\left(r+r^{\prime}\right) l=\lambda_{r+r^{\prime}}$. Under this condition the loop product gives a product

$$
F_{p} C_{p+q}(\mathcal{L} M) \otimes F_{p^{\prime}} C_{p^{\prime}+q^{\prime}}(\mathcal{L} M) \rightarrow F_{p+p^{\prime}} C_{p+q+p^{\prime}+q^{\prime}-d}(\mathcal{L} M) .
$$

This implies the following proposition.

Proposition 2.2 Let $M$ be a $d$-dimensional compact Riemannian manifold that satisfies Condition $(\mathrm{Cl})$. Then, the loop product induces a multiplicative structure on the shifted Morse spectral sequence $\mathbb{E}_{p, q}^{r}(\mathcal{M})(\mathcal{L} M):=E_{p, q+d}^{r}(\mathcal{M})(\mathcal{L} M)$.

Remark Of course the inequality $\lambda_{r}+\lambda_{r^{\prime}} \leq \lambda_{r+r^{\prime}}$ is essential in order to have a multiplicative structure. It would be interesting to investigate the existence of metrics that satisfy this condition, in particular in the case of connected sums of symmetric spaces.

The following Lemma gives the bigraded module structure of $\mathbb{E}_{*, *}^{1}(\mathcal{M})(\mathcal{L} M)$ in function of $H_{*}(M), H_{*}(U M)$ and of $\alpha_{r}$. Where $\alpha_{r}$ is the index of the critical value $\lambda_{r}$ namely the dimension of the fiber of $\mu_{r}^{-} \rightarrow \Sigma_{r}$.

Lemma 2.3 If $M$ is a Riemannian manifold that satisfies Condition (Cl), then for $r>0$ we have isomorphisms of modules

$$
\phi_{r}: H_{*}\left(\mathcal{L} M^{\leq \lambda_{r}}, \mathcal{L} M^{\leq \lambda_{r-1}}\right) \rightarrow H_{*-\alpha_{r}}(U M) .
$$

Proof By retraction along the gradient flow lines of the energy functional the space $\mathcal{L} M^{<\lambda_{r}}$ retracts to $\mathcal{L} M^{\leq \lambda_{r-1}}$. This gives an isomorphism

$$
H_{*}\left(\mathcal{L} M^{\leq \lambda_{r}}, \mathcal{L} M^{\leq \lambda_{r-1}}\right) \rightarrow H_{*}\left(\mathcal{L} M^{\leq \lambda_{r}}, \mathcal{L} M^{<\lambda_{r}}\right) .
$$


The right term is equal to $H_{*}\left(\mathcal{L} M^{\leq \lambda_{r}}, \mathcal{L} M^{\leq \lambda_{r}}-\Sigma_{r}\right)$. By excision isomorphism, it is isomorphic to $H_{*}\left(D_{\alpha_{r}}\left(\Sigma_{r}\right), S_{\alpha_{r}-1}\left(\Sigma_{r}\right)\right)$. Next, $H_{*}\left(D_{\alpha_{r}}\left(\Sigma_{r}\right), S_{\alpha_{r}-1}\left(\Sigma_{r}\right)\right)$ is isomorphic to $H_{*-\alpha_{r}}(U M)$ by the Thom isomorphism. Since $M$ satisfies Condition $(\mathrm{Cl})$, then $\Sigma_{r}$ is diffeomorphic to $U M$. The composition of these isomorphisms gives the required isomorphism

$$
\phi_{r}: H_{*}\left(\mathcal{L} M^{\leq \lambda_{r}}, \mathcal{L} M^{\leq \lambda_{r-1}}\right) \rightarrow H_{*-\alpha_{r}}(U M) .
$$

Corollary 2.4 The bigraded module $\bigoplus_{p, q \geq 0} E_{p, q}^{1}(\mathcal{M})(\mathcal{L} M)$ is isomorphic to $H_{q}(M)$ if $p=0$ and to $\bigoplus_{p \geq 1, q \geq 0} H_{p+q-\alpha_{p}}(U M)$ if $p \geq 1$.

Proof This is direct application of Theorem 2.1 and of Lemma 2.3.

The multiplicative structure In what follows we reformulate Corollary 12.7 of Theorem 12.5 of [10]. We introduce a bigraded algebra $\mathcal{A}_{*, *}$. As bigraded module, $\mathcal{A}_{*, *}$ is a regraduation by a translation of $d$ of the bigraded module of Lemma 2.3. We set

$$
\begin{aligned}
& \mathcal{A}_{0, q}:=\mathbb{H}_{q}(M), \\
& \mathcal{A}_{p, q}:=\mathbb{H}_{p+q-\alpha_{p}}(U M)\left\langle T^{p}\right\rangle \text { if } p>0
\end{aligned}
$$

where $T$ is an element of bidegree $\left(1, \alpha_{1}+d-2\right)$. Moreover we suppose that we have Bott's iteration formula:

$$
\alpha_{p}=p \alpha_{1}+(p-1)+(d-1) .
$$

The multiplicative structure of $\mathcal{A}_{*, *}$ is given by:

(1) the intersection algebra $\mathbb{H}_{*}(M)$ if $p=0$,

(2) the algebra $\mathbb{H}_{p+q-\alpha_{p}}(U M)[T]_{\geq 1}$ of polynomials of degree $\geq 1$ in $T$ when $p>0$,

(3) the products involving an element of $\mathbb{H}_{*}(M)$, and an element of the algebra $\mathbb{H}_{p+q-\alpha_{p}}(U M)[T]_{\geq 1}$, which are given by a topological $\mathbb{H}_{*}(M)$-module structure on $\mathbb{H}_{*}(U M)$. We denote it by

$$
m: \mathbb{H}_{s}(M) \otimes \mathbb{H}_{t}(U M) \rightarrow \mathbb{H}_{s+t}(U M) .
$$

This structure is given by the map

$$
m=\widehat{\delta_{M}} ! \circ \times
$$

where $x$ is the cross product

$$
H_{i}(M) \otimes H_{j}(U M) \rightarrow H_{i+j}(M \times U M)
$$


and $\widehat{\delta_{M}}$ ! is the Gysin map associated to $\widehat{\delta_{M}}$ defined using the pullback map

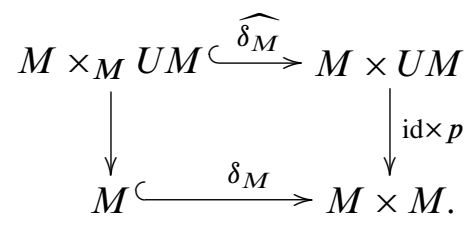

At the homology level we have the morphism

$$
\begin{aligned}
\mathbb{H}_{u}(M) \otimes \mathbb{H}_{v}(U M) T^{k} & \rightarrow \mathbb{H}_{u+v}(U M) T^{k} \\
x \otimes y T^{k} & \mapsto m(x, y) T^{k} .
\end{aligned}
$$

Theorem 2.5 Let $M$ be a $d$-dimensional compact Riemannian manifold that satisfies Condition $(C l)$. As algebra, $\mathbb{E}_{*, *}^{1}(\mathcal{M})(\mathcal{L} M)$ is isomorphic to $\mathcal{A}_{*, *}$. In other words, the multiplicative structure on the shifted Morse spectral sequence $\mathbb{E}_{p, q}^{r}(\mathcal{M})(\mathcal{L} M):=$ $E_{p, q+d}^{r}(\mathcal{M})(\mathcal{L} M)$ is given at the $E^{1}$-level by

$$
\mathbb{E}_{*, *}^{1}(\mathcal{M})(\mathcal{L} M)=\mathbb{H}_{*}(M) \oplus \mathbb{H}_{*}(U M)[T]_{\geq 1}
$$

The bidegree of $T$ is $\left(1, \alpha_{1}+d-2\right)$, elements of $\mathbb{H}_{*}(M)$ and of $\mathbb{H}_{*}(U M)$ are of bidegree $(0, *)$. We have

$$
\mathbb{E}_{0, q}^{1}(\mathcal{M})(\mathcal{L} M)=\mathbb{H}_{q}(M)
$$

and for $p \geq 1$,

$$
\mathbb{E}_{p, q}^{1}(\mathcal{M})(\mathcal{L} M)=\mathbb{H}_{q-p \alpha_{1}}(U M)\left\langle T^{p}\right\rangle .
$$

The multiplication between the 0-th column and the others is induced by the $\mathbb{H}_{*}(M)$ module structure on $\mathbb{H}_{*}(U M)$.

Proof Corollary 2.4 tells us that $\mathbb{E}_{*, *}^{1}(\mathcal{M})(\mathcal{L} M)$ and $\mathcal{A}_{*, *}$ are isomorphic as modules. It remains to prove that there multiplicative structures are isomorphic. This is a direct consequence of the diagram on page 39 of Hingston and Goresky [10]; see also Laudenbach [13]. The diagonal map on $U M$ factorizes as

$$
U M \stackrel{\delta_{S}}{\longrightarrow} U M \times_{M} U M \stackrel{\delta_{M}}{\longrightarrow} U M \times U M
$$


For simplicity, we denote $\mathcal{L} M^{\leq \lambda_{r}}$ by $\Lambda_{r}$. At the level of homology we have the following commutative diagram:

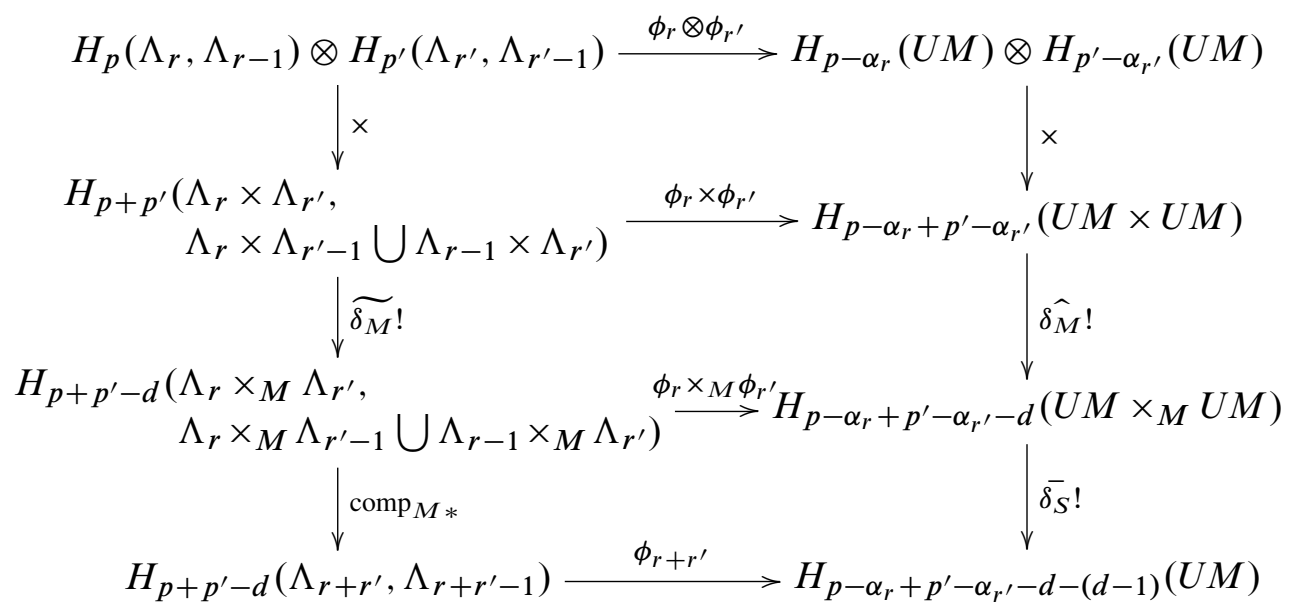

We recall Bott's iteration formula [4]:

$$
\alpha_{r+r^{\prime}}=\alpha_{r}+\alpha_{r^{\prime}}+d-1
$$

Then we have

$$
H_{p+p^{\prime}-d}\left(\Lambda_{r+r^{\prime}}, \Lambda_{r+r^{\prime}-1}\right) \stackrel{\phi_{r+r^{\prime}}}{\longrightarrow} H_{p+p^{\prime}-d-\alpha_{r+r^{\prime}}}(U M) .
$$

Thus we have for $p \geq 1$, the multiplicative identification

$$
\mathbb{E}_{p, q}^{1}(\mathcal{M})(\mathcal{L} M)=\mathbb{H}_{q-p \alpha_{1}}(U M)\left\langle T^{p}\right\rangle .
$$

For $p=0$, we observe that $\mathbb{H}_{*}\left(\mathcal{L} M^{\leq 0}, \mathcal{L} M^{\leq-1}\right)$ with the loop product is in fact $\mathbb{H}_{*}(M)$ with the intersection product. This allows us to identify the 0 -th column:

$$
\mathbb{E}_{0, q}^{1}(\mathcal{M})(\mathcal{L} M)=\mathbb{H}_{q}(M) .
$$

And for $p$ or $p^{\prime}$ equal to zero, we get the $\mathbb{E}_{0, *}^{1}(\mathcal{M})$-module structure given by $m$ on $\mathbb{E}_{*, *}^{1}(\mathcal{M})(\mathcal{L} M)$.

\subsection{The example of spheres}

Let us illustrate the preceding theorem, we consider the case of spheres. The computation of the Betti numbers was done by Bott in [3]. Bott's method was used by Ziller to completely determine the homology of free loop spaces of symmetric spaces of rank one [20]. Ziller proved that the Morse spectral sequence collapses at the $E_{1}$-term. 
We recall that on the $n$-sphere together with its standard Riemannian structure, the closed geodesics are represented by nondegenerate critical manifolds of two types:

- $\operatorname{Geod}_{0}=S^{n}$ corresponding to constant loops,

- $\operatorname{Geod}_{k}=U S^{n}$ corresponding to the great circles starting to a unit vector $u \in U S^{n}$ and traversing that circle $k$ times.

The indices for $k>0$ are given by the formula

$$
\operatorname{index}\left(\operatorname{Geod}_{k}\right)=(2 k-1)(n-1) .
$$

We consider the Morse spectral sequence for spheres. The $E^{1}$-term is generated by elements on $E_{0, *}^{1}$ and on $E_{1, *}^{1}$. As we have a spectral of algebra the differential $d^{1}$ is a derivation, thus to compute $d^{1}$ it suffices to compute it on $E_{0, *}^{1}$ and $E_{1, *}^{1}$. Thanks to inclusion of the constant loops in $\mathcal{L} S^{n}$, which is a section of the evaluation map, we deduce that all the elements of $E_{0, *}^{1}$ must survive. We thus have that $d^{1}=0$. As algebra we find that $E^{1}=E^{2}$ and for degree reasons the derivation satisfy $d^{r}=0$ for $r>1$. Finally we have $E^{\infty}=E^{1}$.

In the even case, there is no extension issue. In the odd case, one is left with one multiplicative extension issue, but one can solve it by using the intersection morphism

$$
\mathbb{H}_{*}\left(L S^{n}\right) \rightarrow H_{*}\left(\Omega S^{n}\right) .
$$

At the end we recover that

$$
\mathbb{H}_{*}\left(\mathcal{L} S^{n}\right) \cong\left\{\begin{array}{cl}
\Lambda(a) \otimes \mathbb{Z}[u] & \text { for } n \text { odd } \\
(\Lambda(b) \otimes \mathbb{Z}[a, v]) /\left(a^{2}, a b, 2 a v\right) & \text { for } n \text { even. }
\end{array}\right.
$$

In the Morse spectral sequence we have $a \in \mathbb{E}_{0,-n}^{\infty}, b \in \mathbb{E}_{1,-2}^{\infty}, u \in \mathbb{E}_{1, n-2}^{\infty}$ and $v \in \mathbb{E}_{1,2 n-3}^{\infty}$.

It may be interesting to notice that the energy filtration on spheres corresponds to the filtration of algebraic loops on spheres by their polynomial degree [2]; the same fact holds for complex projective spaces.

\subsection{Morse-Serre spectral sequences}

In this section we define a fiberwise version of the preceding spectral sequence. This spectral sequence is associated to a fibration

$$
\text { Fiber } \rightarrow E \rightarrow B
$$


where we have a Morse filtration of the base $B$ (in our case the length filtration of a loop space) and we use it to filter the total space $E$. In the classical case, where we have a smooth fiber bundle

$$
\text { Fiber } \rightarrow N \rightarrow M
$$

of closed oriented compact manifolds and a Morse function $f: M \rightarrow \mathbb{R}$, we recover the Leray-Serre spectral sequence of the fiber bundle. This approach is very close in spirit to the treatment of Floer homology of families; see for example M Hutchings' paper [12].

2.4.1 Definition of the spectral sequence Let $M$ be a $d$-dimensional compact oriented Riemannian manifold. Assume that the critical points of the energy function on $\mathcal{L} M$ are collected on compact critical manifolds. We also assume that each of these critical manifolds satisfy the Bott nondegeneracy condition.

Let $p: X \rightarrow M$ be a Serre fibration (resp. a fiber bundle) over $M$ with fiber $F$ such that $F$ is a closed smooth manifold of finite dimension $f$. Let us consider the fibration (the fiber bundle) $\pi:=\mathcal{L} p: \mathcal{L} X \rightarrow \mathcal{L} M$ with fiber $\mathcal{L} F$. As in the preceding sections, we filter $\mathcal{L} M$ by the critical values $\lambda_{r}$ of the energy functional. This filtration induces a filtration on $\mathcal{L} X$ that we denote by $\mathcal{L} X_{\leq \lambda_{r}}$ defined by the following pullback diagram.

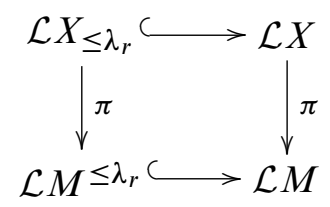

We define

$$
\widetilde{\Sigma_{r}}:=\left\{\gamma \in \mathcal{L} X / E(\pi(\gamma))=\lambda_{r} \text { and } d E(\pi(\gamma))=0\right\} .
$$

We remark that $\widetilde{\Sigma_{r}}$ is obtained from $\pi$ and $\Sigma_{r}$ by the pullback diagram

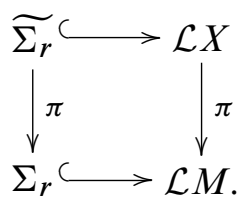

Moreover for the sake of simplicity we suppose that these critical sets are connected. By pulling back over $T \mathcal{L} M$, we define $\widetilde{\mu_{p}^{-}}$in the following way. Let $\widetilde{T \mathcal{L} X}$ be the 
space defined by the pullback diagram

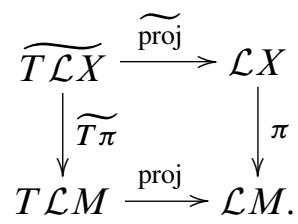

Where proj is the canonical projection of $T \mathcal{L} M$. Then, since

$$
\left.T \mathcal{L} M\right|_{\Sigma_{r}} \simeq \mu_{r}^{-} \oplus \mu_{r}^{0} \oplus \mu_{r}^{+}
$$

the total space of the bundle

$$
\left.\widetilde{T \mathcal{L} M}\right|_{\widetilde{\Sigma_{r}}} \stackrel{\widetilde{T \pi}}{\longrightarrow} \widetilde{\Sigma_{r}}
$$

splits into three parts

$$
\left.\widetilde{T \mathcal{L} X}\right|_{\widetilde{\Sigma_{r}}} \simeq \widetilde{\mu_{r}^{-}} \oplus \widetilde{\mu_{r}^{0}} \oplus \widetilde{\mu_{r}^{+}}
$$

We have the following commutative diagram:

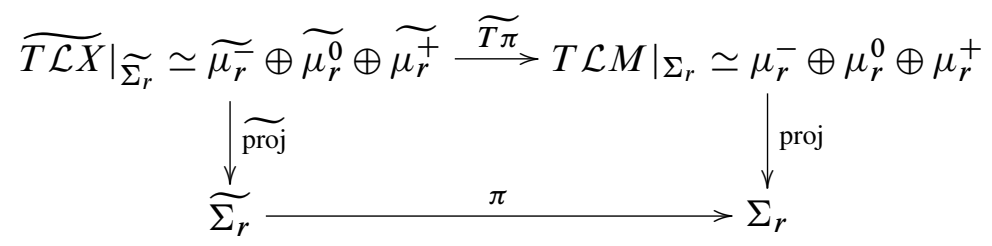

Theorem 2.6 Let $p: X \rightarrow M$ be a fiber bundle over a Riemannian manifold equipped with a metric such that the critical sets of the energy functional on $\mathcal{L} M$ satisfy the Bott nondegeneracy condition. Then the fiberwise energy filtration of $C_{*}(\mathcal{L} X)$ induces a spectral sequence called the Morse-Serre spectral sequence $\left\{E_{*, *}^{r}(\mathcal{M S})(\pi)\right\}_{r \in \mathbb{N}}$ converging to $H_{*}(\mathcal{L} X)$ denoted by

$$
E_{p, q}^{r}(\mathcal{M S})(\pi) \Rightarrow H_{p+q}(\mathcal{L} X)
$$

The $E^{1}$-page is given by

$$
E_{p, q}^{1}(\mathcal{M S})(\pi)=H_{p+q}\left(\mathcal{L} X_{\leq \lambda_{p}}, \mathcal{L} X_{\leq \lambda_{p-1}}\right)
$$

so that $E_{p, q}^{1}(\mathcal{M S})(\pi)$ is isomorphic to the reduced homology $\tilde{H}_{p+q}\left(\operatorname{Th}\left(\widetilde{\mu_{p}^{-}}\right)\right)$. 
Proof We have a pullback square

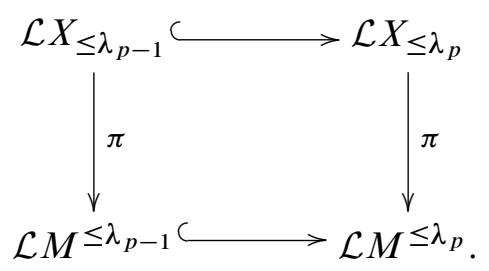

The embedding of $\mathcal{L} X_{\leq \lambda_{p-1}}$ into $\mathcal{L} X_{\leq \lambda_{p}}$ has a normal bundle which is the pullback of the normal bundle of the embedding of $\mathcal{L} M^{\leq \lambda_{p-1}}$ into $\mathcal{L} M^{\leq \lambda_{p}}$. We recall that the space $\mathcal{L} M^{\leq \lambda_{p}} / \mathcal{L} M^{\leq \lambda_{p-1}}$ is homotopy equivalent to the Thom space $\operatorname{Th}\left(\mu_{p}^{-}\right)$ we deduce that $\mathcal{L} X_{\leq \lambda_{p}} / \mathcal{L} X_{\leq \lambda_{p-1}}$ is homotopically equivalent to the Thom space $\operatorname{Th}\left(\widetilde{\mu_{p}^{-}}\right)$.

2.4.2 Compatibility with the loop product We assume that $M$ satisfies Condition $(\mathrm{Cl})$.

The embedding

$$
\begin{aligned}
\delta_{X}: X \hookrightarrow X \times X, & \hookrightarrow X+x) \\
x & \mapsto(x, x)
\end{aligned}
$$

factorizes by the map $\delta_{X}^{1}$ defined by the pullback diagram

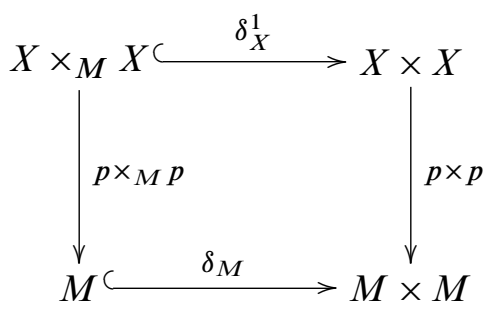

and by the canonical map

$$
\delta_{X}^{2}: X \hookrightarrow X \times_{M} X
$$

induced by the diagonal map on $X$ and the projection on $M$. Thus, we have

$$
\delta_{X}=\delta_{X}^{1} \circ \delta_{X}^{2}
$$

We denote by $\widetilde{\delta_{X}^{i}}$ the pullback of $\delta_{X}^{i}$ over the free loop fibration, $i=1,2$. We remark that $\delta_{X}^{1}$ is a smooth embedding of codimension $\operatorname{dim}(F)$, and we denote it by $f$. 
Let us recall that $F_{p} C_{p+q}(\mathcal{L} X)=C_{p+q}\left(\mathcal{L} X_{\leq \lambda_{p}}\right)$ then the loop product is given by the following composition map.

$$
\begin{aligned}
& C_{p+q}\left(\mathcal{L} X_{\leq \lambda_{p}}\right) \otimes C_{p^{\prime}+q^{\prime}}\left(\mathcal{L} X_{\leq \lambda_{p^{\prime}}}\right) \\
& \downarrow x \\
& C_{p+q+p^{\prime}+q^{\prime}}\left(\mathcal{L} X_{\leq \lambda_{p}} \times \mathcal{L} X_{\leq \lambda_{p^{\prime}}}\right) \\
& \downarrow \widetilde{\delta_{X}^{1}} \text { ! } \\
& C_{p+q+p^{\prime}+q^{\prime}-d}\left(\mathcal{L} X_{\leq \lambda_{p}} \times_{M} \mathcal{L} X_{\leq \lambda_{p^{\prime}}}\right) \\
& \downarrow \widetilde{\delta_{X}^{2}} \text { ! } \\
& C_{p+q+p^{\prime}+q^{\prime}-d-f}\left(\mathcal{L} X_{\leq \lambda_{p}} \times_{X} \mathcal{L} X_{\leq \lambda_{p^{\prime}}}\right) \\
& \downarrow \operatorname{comp}_{X *} \\
& C_{p+q+p^{\prime}+q^{\prime}-d-f}\left(\mathcal{L} X_{\leq \lambda_{p}+\lambda_{p^{\prime}}}\right)=C_{p+q+p^{\prime}+q^{\prime}-d-f}\left(\mathcal{L} X_{\leq \lambda_{p+p^{\prime}}}\right)
\end{aligned}
$$

This gives a multiplicative structure to the $(0, d+f)$-regraded Morse Serre spectral sequence. We have the following proposition:

Proposition 2.7 Let $M$ be a Riemannian compact $d$-dimensional manifold that satisfies Condition (Cl). Let $\pi: X \rightarrow M$ be a fibration over $M$. Then, the loop product induces a multiplicative structure on the regraded Morse Serre spectral sequence $\mathbb{E}_{p, q}^{r}(\mathcal{M S})(\pi):=E_{p, q+d+f}^{r}(\mathcal{M S})(\pi)$.

2.4.3 The first page Now let us focus on the $E^{1}$-page of the Morse-Serre spectral sequence. In order to describe this first page and its multiplicative structure we will introduce a bigraded algebra $\mathcal{A}_{*, *}^{F}$, this bigraded algebra is the fibrewise analogue of the bigraded algebra $\mathcal{A}_{*, *}$ introduced in the preceding sections.

Let $\widetilde{M}$ and $\widetilde{U M}$ the fiberwise analogues of the spaces $M$ and $U M$ :

(a) The space $\widetilde{M}$ is defined as the pullback of the inclusion of constant loops $M \subset$ $\mathcal{L} M$ along the fibration $\pi: \mathcal{L} X \rightarrow L M$. We thus have a fibration $c: \widetilde{M} \rightarrow M$.

(b) The space $\widetilde{U M}$ is defined as the pullback of the map geod: $U M \rightarrow \mathcal{L} M$ which associates to a unit tangent vector $u$ the unique closed geodesic such that $\dot{\gamma}(0)=u$. We also have a fibration $b: \widetilde{U M} \rightarrow U M$.

We define a multiplicative structure on $\mathbb{H}_{*}(\widetilde{U M}):=H_{*+d+d-1+f}(\widetilde{U M})$ that mixes the intersection product on $U M$ and the loop product on the fiber $L F$ of $\pi$. This 
product is associated to the following commutative diagram:

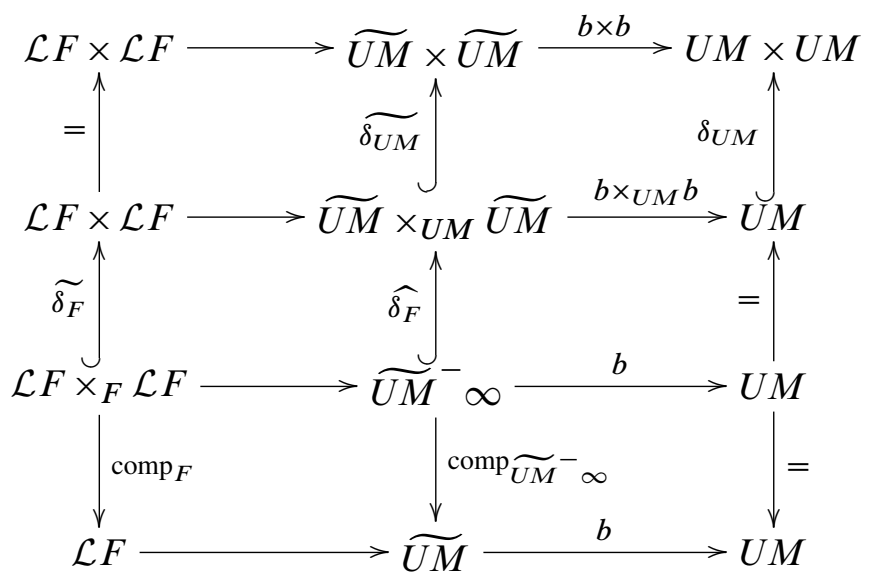

where $\widetilde{U M}^{\infty}$ denotes the composable loops of $\widetilde{U M}$. The product is given by the composition

$$
\operatorname{comp} \widetilde{U M}^{\infty} \widehat{\circ} \widehat{\delta_{F}} ! \circ \widetilde{\delta_{U M}} ! \circ \times
$$

In the same way we a define a multiplicative structure for the homology of $\widetilde{M}$ by considering it as the total space of the fibration $c$. This provides $\mathbb{H}_{*}(\widetilde{M}):=H_{*+d+f}(\widetilde{M})$ with a structure of algebra.

Furthermore, there is a structure of $\mathbb{H}_{*}(\widetilde{M})$-module on $\mathbb{H}_{*}(\widetilde{U M})$. This structure comes from the commutative diagram

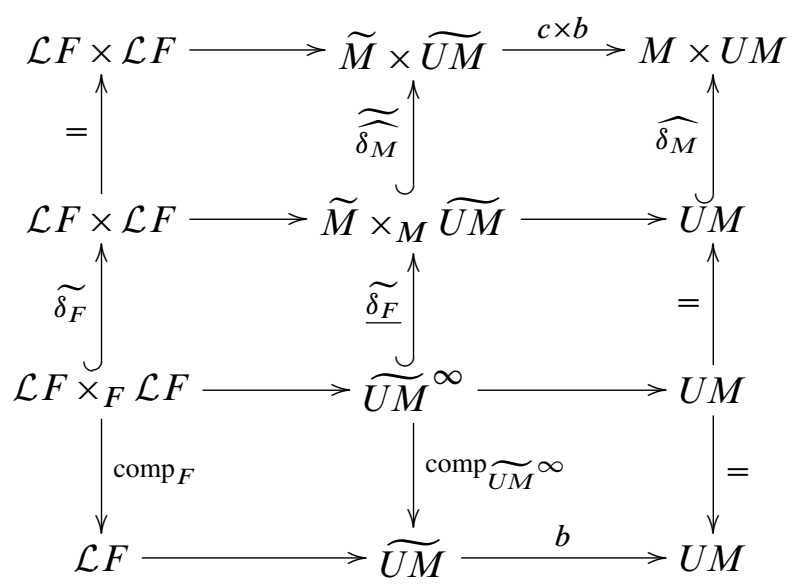


where $\widehat{\delta_{M}}$ denotes the inclusion

$$
\begin{aligned}
\widehat{\delta_{M}}: U M & \hookrightarrow M \times U M \\
x & \mapsto(\operatorname{proj}(x), x)
\end{aligned}
$$

with proj: $U M \rightarrow M$ is the canonical projection. The module structure

$$
\tilde{m}: \mathbb{H}_{*}(\widetilde{M}) \otimes \mathbb{H}_{*}(\widetilde{U M}) \rightarrow \mathbb{H}_{*}(\widetilde{U M})
$$

is given by

$$
\tilde{m}=\operatorname{comp}_{\widetilde{U M}} \infty \circ \stackrel{\widetilde{\delta_{F}}}{\circ} \circ \widetilde{\widetilde{\delta_{M}}} ! \circ \times
$$

Definition 2.8 These products on $\mathbb{H}_{*}(\widetilde{U M})$ and on $\mathbb{H}_{*}(\widetilde{M})$ are called the fiberwise intersection loop product. The module structure $\tilde{m}$ of $\mathbb{H}_{*}(\widetilde{M})$ on $\mathbb{H}_{*}(\widetilde{U M})$ is called the fiberwise loop product module structure. The bigraded algebra $\mathcal{A}_{*, *}^{F}$ is defined by

(1) $\mathcal{A}_{0, *}^{F}=\mathbb{H}_{*}(\widetilde{M})$,

(2) $\mathcal{A}_{p, q}^{F}=\mathbb{H}_{q-p \alpha_{1}}(\widetilde{U M})\left\langle T^{p}\right\rangle$ with $T$ of bidegree $\left(1, \alpha_{1}+d-2\right)$ for $p>0$.

The multiplicative structure is given by the fiberwise loop product.

Let us give the main Theorem of this section. This theorem will be one of the main computational tool of this paper.

Theorem 2.9 Let $M$ be a $d$-dimensional compact oriented Riemannian manifold that satisfies Condition (Cl). Let $p: X \rightarrow M$ be a fibration over $M$. The $E^{1}$-page of the regraded Morse-Serre spectral sequence $\mathbb{E}_{p, q}^{r}(\mathcal{M S})(\pi):=E_{p, q+d+f}^{r}(\mathcal{M S})(\pi)$ is isomorphic as a bigraded algebra to $\mathcal{A}_{*, *}^{F}$.

Proof For $r \geq 1$, we define

$$
\widetilde{\phi_{r}}: H_{*}\left(\mathcal{L} X_{\leq \lambda_{r}}, \mathcal{L} X_{\leq \lambda_{r-1}}\right) \rightarrow H_{*-\alpha_{r}}(\widetilde{U M})
$$

by composition of the Thom isomorphism

$$
\mathcal{T}: H_{*}\left(\mathcal{L} X_{\leq \lambda_{r}}, \mathcal{L} X_{\leq \lambda_{r-1}}\right) \rightarrow H_{*-\alpha_{r}}\left(\widetilde{\Sigma_{r}}\right)
$$


with the identification of $H_{*-\alpha_{r}}\left(\widetilde{\Sigma_{r}}\right)$ with $H_{*-\alpha_{r}}(\widetilde{U M})$. Let us denote $\mathcal{L} X_{\leq \lambda_{r}}$ by $\Lambda_{r}$, the proof follows from the following commutative diagram:

$$
\begin{aligned}
& H_{p}\left(\Lambda_{r}, \Lambda_{r-1}\right) \otimes H_{p^{\prime}}\left(\Lambda_{r^{\prime}}, \Lambda_{r^{\prime}-1}\right) \stackrel{\widetilde{\phi_{r}} \otimes \widetilde{\phi_{r}}}{\longrightarrow} H_{p-\alpha_{r}}(\widetilde{U M}) \otimes H_{p^{\prime}-\alpha_{r^{\prime}}}(\widetilde{U M}) \\
& H_{p+p^{\prime}}\left(\Lambda_{r} \times \Lambda_{r^{\prime}}, \quad \widetilde{\phi_{r}} \times \widetilde{\phi_{r}} \underset{ }{ } \quad \downarrow\right. \\
& \begin{aligned}
H_{p+p^{\prime}}\left(\Lambda_{r} \times \Lambda_{r^{\prime}},\right. \\
\left.\Lambda_{r} \times \Lambda_{r-1} \cup \Lambda_{r-1} \times \Lambda_{r^{\prime}}\right)
\end{aligned} \stackrel{\widetilde{\phi_{r}} \times \widetilde{\phi_{r}}}{\longrightarrow} H_{p+p^{\prime}-\alpha_{r}-\alpha_{r^{\prime}}}(\widetilde{U M} \times \widetilde{U M}) \\
& \downarrow \widetilde{\delta_{X} !} \quad \sim \widehat{\delta_{F} ! \widetilde{\delta_{U M}} !}
\end{aligned}
$$

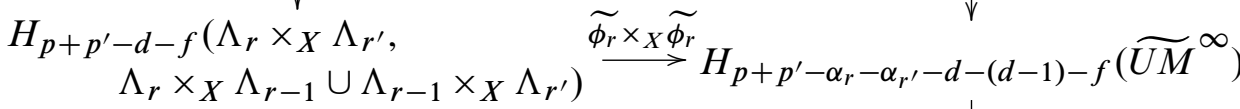

$$
\begin{aligned}
& \downarrow_{p+p^{\prime}-d-f}\left(\Lambda_{r+r^{\prime}}\right) \stackrel{\operatorname{comp}_{X *}}{\widetilde{\phi_{r+r^{\prime}}}} \longrightarrow H_{p+p^{\prime}-\alpha_{r}-\alpha_{r^{\prime}}-d-(d-1)-f}(\widetilde{U M})
\end{aligned}
$$

The last line can be rewritten

$$
H_{p+p^{\prime}-d-f}\left(\Lambda_{r+r^{\prime}}, \Lambda_{r+r^{\prime}-1}\right) \rightarrow H_{p+p^{\prime}-\alpha_{r+r^{\prime}}-d-(d-1)-f}(\widetilde{U M}) .
$$

For $p=0$, we notice that $\mathbb{H}_{*}\left(\mathcal{L} X_{\leq 0}, \mathcal{L} X_{\leq-1}\right)$ with the loop product is in fact $\mathbb{H}_{*}(\widetilde{M})$ with the fiberwise intersection loop product. This allows the identification of the 0 -th column:

$$
\mathbb{E}_{0, q}^{1}(\mathcal{M S})(\pi)=\mathbb{H}_{q}(\widetilde{M})
$$

\section{The 0-th column of the spectral sequence}

In this section we begin the computation of $\mathbb{H}_{*}\left(\operatorname{Imm}\left(S^{1}, S^{n}\right)\right)$. By using HirschSmale theory we replace the space $\operatorname{Imm}\left(S^{1}, S^{n}\right)$ by the loop space $\mathcal{L} U S^{n}$ and we consider the fiber bundle

$$
\mathcal{L} S^{n-1} \rightarrow \mathcal{L} U S^{n} \rightarrow \mathcal{L} S^{n}
$$

associated to the unit tangent bundle

$$
S^{n-1} \rightarrow U S^{n} \rightarrow S^{n}
$$

We filter the space $\mathcal{L} U S^{n}$ by the length filtration of the base $\mathcal{L} S^{n}$ and compute the associated spectral sequence. We notice that the 0 -th column of the $E^{1}$-term of this spectral sequence is the homology of the space of vertical loops of $\mathcal{L} U S^{n}$. Vertical loops are the loops of $U S^{n}$ constant on $S^{n}$. We denote this space by $\mathcal{L} U S_{v}^{n}$. 


\subsection{The even case}

Proposition 3.1 The algebra $\mathbb{H}_{*}\left(\mathcal{L} U S_{v}^{2 n}\right)$ is isomorphic to

$$
\mathbb{Z}[a, b, c] /\left(b^{2}, 2 b, a^{2}, a b\right)
$$

with $\operatorname{deg}(a)=-4 n+1, \operatorname{deg}(b)=-2 n$ and $\operatorname{deg}(c)=2 n-2$.

Proof By Theorem 2.9 we have the isomorphism $\mathbb{E}_{0, *}^{1}(\mathcal{M S})(\pi) \cong \mathbb{H}_{*}\left(\widetilde{S^{2 n}}\right)$. Let us determine the $(2 n, 2 n+1)$-regraded Serre spectral sequence $\mathbb{E}_{*, *}^{*}(c)$ associated to the fibration $c: \widetilde{S^{2 n}} \rightarrow S^{2 n}$ with fiber $\mathcal{L} S^{2 n-1}$. This spectral sequence is multiplicative and

$$
\mathbb{E}_{*, *}^{2}(c)=\mathbb{H}_{*}\left(S^{2 n} ; \mathbb{H}_{*}\left(\mathcal{L} S^{2 n-1}\right)\right)=\mathbb{H}_{*}\left(S^{2 n}\right) \otimes \mathbb{H}_{*}\left(\mathcal{L} S^{2 n-1}\right)
$$

since $\mathbb{H}_{*}\left(S^{2 n}\right)$ has no torsion. Then, $\mathbb{E}_{*, *}^{2}(c)$ is isomorphic to

$$
\mathbb{Z}\left[x_{-2 n}\right] / x_{-2 n}^{2} \otimes \mathbb{Z}\left[u_{2 n-2}, y_{-2 n+1}\right] / y_{-2 n+1}^{2} .
$$

In the diagram in Figure 1 and on the others of this paper, the symbols $\mathrm{X}$ represent the abelian group $\mathbb{Z}$ and the symbols $\mathrm{O}$ the field $\mathbb{Z} / 2 \mathbb{Z}$. The generators of algebra are represented by squares.

Since this spectral sequence is a spectral sequence of algebra, we only need to compute the differentials on the generators. For reasons of degree, the only possible nonzero differential is $d_{2 n}\left(y_{-2 n+1}\right)$. The map ev(0): $\mathcal{L} U S_{\leq 0}^{2 n} \rightarrow U S^{2 n}$ has a section so that $\mathbb{H}_{-2 n}\left(U S^{2 n}\right)$ is isomorphic to $\mathbb{H}_{-2 n}\left(\widetilde{S^{2 n}}\right)$ namely $\mathbb{Z} / 2 \mathbb{Z}$. This implies that $d_{2 n}\left(y_{-2 n+1}\right)=2 x_{-2 n}$.

For degree reasons and by position in the filtration, there is neither linear nor multiplicative extension issues so that

$$
\mathbb{H}_{*}\left(\widetilde{S^{2 n}}\right) \cong \mathbb{Z}\left[w_{-4 n+1}, x_{-2 n}, u_{2 n-2}\right] /\left(w_{-4 n+1}^{2}, w_{-4 n+1} x_{-2 n}, x_{-2 n}^{2}, 2 x_{-2 n}\right)
$$

where $w_{-4 n+1}:=x_{-2 n} y_{-2 n+1}$. We denote by $a, b$ and $c$ respectively the image of $w_{-4 n+1}, x_{-2 n}$ and $u_{2 n-2}$ under the isomorphism of graded algebra from $\mathbb{H}_{*}\left(\widetilde{S^{2 n}}\right)$ to $\mathbb{E}_{0, *}^{1}(\mathcal{M S})(\pi)$. The degrees are the same namely $\operatorname{deg}(a)=-4 n+1, \operatorname{deg}(b)=-2 n$ and $\operatorname{deg}(c)=2 n-2$.

\subsection{The odd case}

Proposition 3.2 The algebra $\mathbb{H}_{*}\left(\mathcal{L} U S_{v}^{2 n+1}\right)$ is isomorphic to

$$
\mathbb{Z}[\alpha, \beta, \gamma, \delta] /\left(\alpha^{2}, \gamma^{2}, \beta^{2}, 2 \beta \delta\right)
$$

with $\operatorname{deg}(\alpha)=-2 n-1, \operatorname{deg}(\beta)=-2 n, \operatorname{deg}(\gamma)=-1$ and $\operatorname{deg}(\delta)=4 n-2$. 


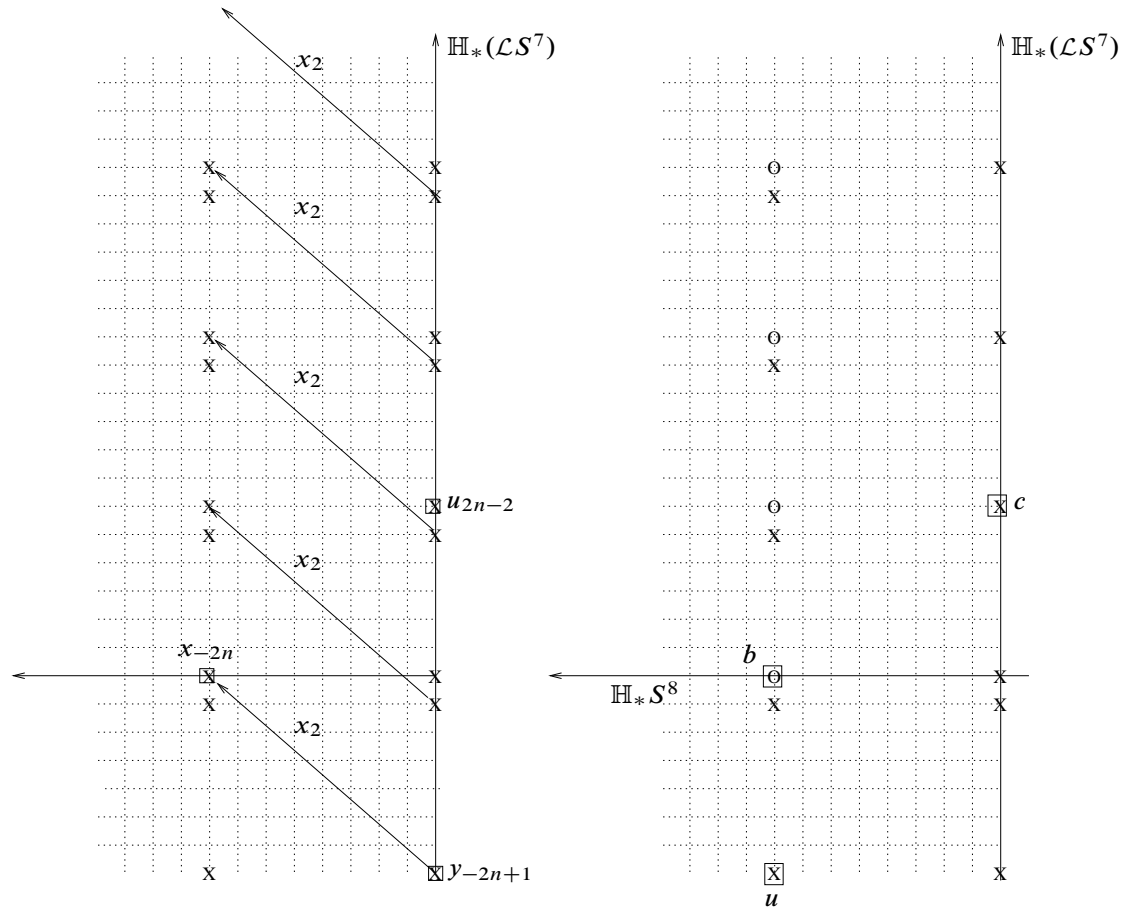

Figure 1

Proof As in the preceding subsection, we compute $\mathbb{E}_{0, *}^{1}(\mathcal{M S})(\pi)$ by computing the $(2 n+1,2 n)$-regraded Serre spectral sequence associated to the fibration $c: S^{2 n+1} \rightarrow$ $S^{2 n+1}$. See Figure 2 .

In this case, the spectral sequence collapses at the $E^{2}$-level because the fibration $\operatorname{ev}(o): \mathcal{L} U S_{\leq 0}^{2 n} \rightarrow U S^{2 n}$ admits a section and so $\mathbb{H}_{-2 n-1}\left(\widehat{S^{2 n+1}}\right) \simeq \mathbb{Z}$. For reasons of degree, there are no extension issues.

\section{Computation of $\mathbb{H}_{*}\left(\mathcal{L} U S^{2 n}\right), n \geq 2$}

Strategy of the proof (1) We begin by computing $\mathbb{H}_{*}\left(\widetilde{U S^{2 n}}\right)$ in Section 4.1, we will get $\mathbb{E}_{1, *}^{1}(\mathcal{M S})(\pi)$. We do it by computing the Serre spectral sequence associated to the fibration $b: \widetilde{U S^{2 n}} \rightarrow U S^{2 n}$.

(2) Then, we compute in Section 4.2 the $\mathbb{H}_{*}\left(\widetilde{S^{2 n}}\right)$-module structure on $\mathbb{H}_{*}\left(\widetilde{U S^{2 n}}\right)$. This will give the multiplicative structure of the first page of the Morse-Serre spectral sequence $\mathbb{E}_{*, *}^{1}(\mathcal{M S})(\pi)$. 


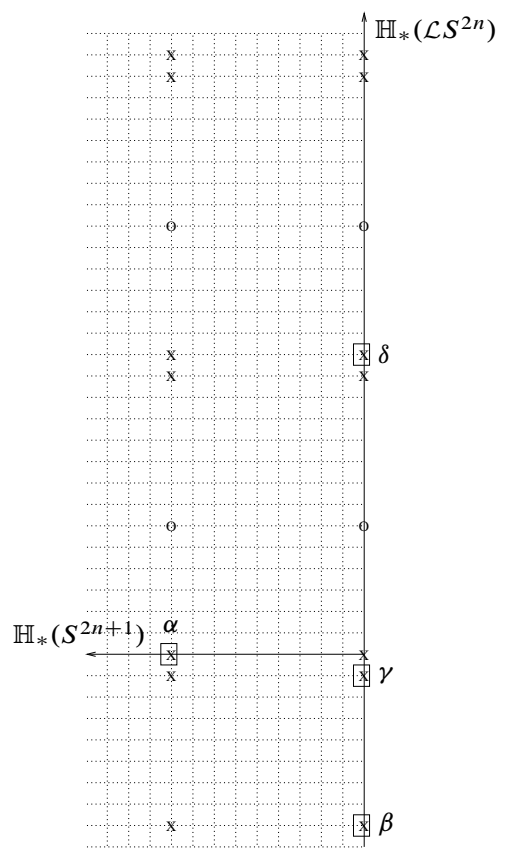

Figure 2

(3) Next, in Section 4.4, we compute the $E^{\infty}$-page of the Cohen-Jones-Yan spectral sequence (that is also the $E^{2}$-page). This gives all the differentials on $\mathbb{E}_{*, *}^{1}(\mathcal{M S})(\pi)$ and leads to $\mathbb{E}_{*, *}^{\infty}(\mathcal{M S})(\pi)$.

(4) Finally, we compare the $E^{\infty}$-page of the two spectral sequences and we solve the linear extension issues and the multiplicative extension issues.

The different stages of the proof are illustrated by pictures representing the different spectral sequences for $n=4$ namely in the case $U S^{8}$.

\subsection{The columns of the Morse-Serre spectral sequence: $\mathbb{E}_{p, *}^{1}(\mathcal{M S})(\pi)$, $p \geq 1$}

In this subsection, we compute the $p$-th columns of the Morse-Serre spectral sequence of $U S^{2 n}$ for $p \geq 1$.

Proposition 4.1 The subalgebra $\mathbb{E}_{p, *}^{1}(\mathcal{M S})(\pi), p \geq 1$, is isomorphic to the algebra

$$
\mathbb{Z}[i, j, k, l] /\left(i^{2}, j^{2}, 2 j, i j, k^{2}\right)[T]_{\geq 1}
$$

with $\operatorname{deg}(i)=-4 n+1, \operatorname{deg}(j)=-2 n, \operatorname{deg}(k)=-2 n+1, \operatorname{deg}(l)=2 n-2$ and $\operatorname{bideg}(T)=(1,4 n-3)$. 
Proof It suffices to compute $\mathbb{E}_{1, *}^{1}(\mathcal{M S})(\pi)$, the other columns are isomorphic up to a shifting and are given by multiplication by $T$.

We begin by applying Theorem 2.9. We first need to compute $\mathbb{H}_{*}\left(\widetilde{U S^{2 n-1}}\right)$. Let us prove that the algebra $\mathbb{H}_{*}\left(\widetilde{U S^{2 n-1}}\right)$ is isomorphic to

$$
\mathbb{Z}\left[x_{-4 n+1}, z_{-2 n}, u_{2 n-2}, y_{-2 n+1}\right] /\left(x_{-4 n+1}^{2}, 2 z_{-2 n}, z_{-2 n}^{2}, y_{-2 n+1}^{2}\right) .
$$

In order to proceed let us compute the $(4 n-1,2 n-1)$-regraded Serre spectral sequence associated to the fibration $b: \widetilde{U S^{2 n}} \rightarrow U S^{2 n}$ with fiber $\mathcal{L} S^{2 n-1}$. We have

$$
\mathbb{E}_{*, *}^{2}(b) \cong \mathbb{H}_{*}\left(U S^{2 n} ; \mathbb{H}_{*}\left(L S^{2 n-1}\right)\right) .
$$

Since $\mathbb{H}_{*}\left(\mathcal{L} S^{2 n-1}\right)$ has no torsion, we also have

$$
\mathbb{H}_{*}\left(U S^{2 n} ; \mathbb{H}_{*}\left(\mathcal{L} S^{2 n-1}\right)\right) \cong \mathbb{H}_{*}\left(U S^{2 n}\right) \otimes \mathbb{H}_{*}\left(\mathcal{L} S^{2 n-1}\right) .
$$

Namely this algebra is isomorphic to

$$
\mathbb{Z}\left[x_{-4 n+1}, z_{-2 n}, u_{2 n-2}, y_{-2 n+1}\right] /\left(x_{-4 n+1}^{2}, 2 z_{-2 n}, z_{-2 n}^{2}, y_{-2 n+1}^{2}\right) .
$$

On the spectral sequence in Figure 3 and on the others of the article, the symbol $\mathrm{O}$ represents the abelian group $\mathbb{Z} / 2 \mathbb{Z}$.

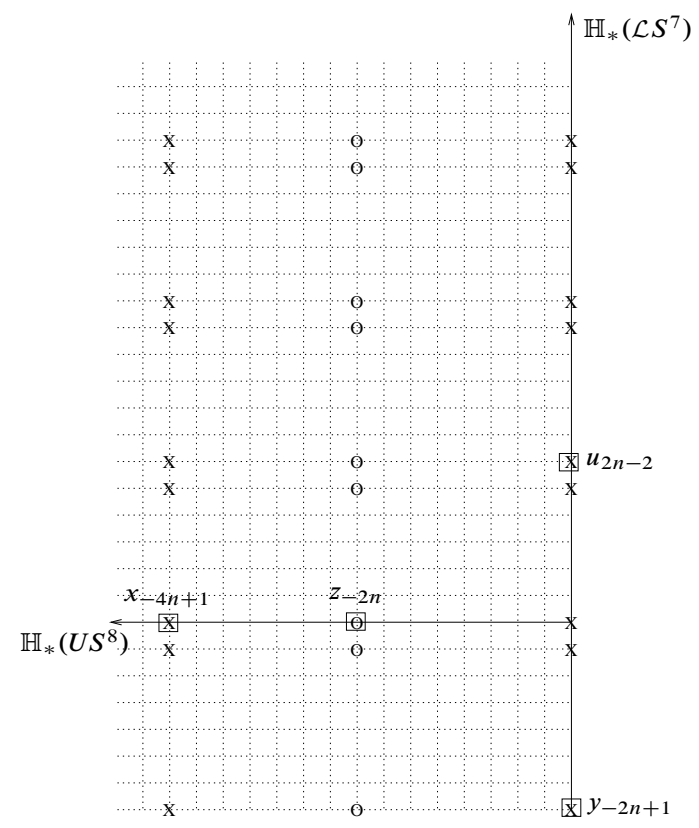

Figure 3 
Let us prove that this spectral sequence collapses at the $E^{2}$-level. We first notice that the fibration $\approx: \widetilde{\Sigma_{1}} \rightarrow \Sigma_{1}$ admits a section. This section is the map

$$
\begin{aligned}
s: \Sigma_{1} & \rightarrow \widetilde{\Sigma_{1}} \\
\gamma & \mapsto\left(\gamma, \frac{\dot{\gamma}}{\|\dot{\gamma}\|}\right) .
\end{aligned}
$$

By the bundle isomorphism $\psi$ of Proposition 2.7, this proves that $b: \widetilde{U S^{2 n}} \rightarrow U S^{2 n}$ admits a section. Then, for degree reasons, the other differentials vanish. To achieve the proof we need to solve the extension issues. The only extension issue is the product $z_{-2 n} y_{-2 n+1}$ which is defined modulo $\mathbb{Z}<x_{-4 n+1}>$. But $z_{-2 n}$ is of 2-torsion, then $z_{-2 n} y_{-2 n+1}$ is also of 2 -torsion. Then the component of $z_{-2 n} y_{-2 n+1}$ is zero. The same argument works for all products $z_{-2 n} y_{-2 n+1} u_{2 n-2}^{k}, k \in \mathbb{N}$.

We represent the first page of the Morse-Serre spectral sequence $\mathbb{E}_{*, *}^{1}(\mathcal{M S})(\pi)$ in Figure 4.

\subsection{The $\mathbb{E}_{0, *}^{1}(\mathcal{M S})(\pi)$-module structure on $\mathbb{E}_{1, *}^{1}(\mathcal{M S})(\pi)$}

Proposition 4.2 The structure of $\mathbb{H}_{*}\left(\widetilde{S^{2 n}}\right)$-module on $\mathbb{H}_{*}\left(\widetilde{U S^{2 n}}\right)$ is entirely described by the following products: $c . i=l i, c . j=l j, c . k=l k, c . l=l^{2}, b . k=j k$, $b . l=l j, a . l=k l j$. The other products are zero.

Proof It follows from the commutative diagram of Figure 1 that we have a $\mathbb{E}_{*, *}^{*}(c)$ module structure on $\mathbb{E}_{*, *}^{*}(b)$. Then, we will compute this module structure using these spectral sequences. At the $E^{2}$-level, this module structure is given by the Chas-Sullivan loop product on the fiber and by the $\mathbb{H}_{*}\left(S^{2 n}\right)$-module structure on $\mathbb{H}_{*}\left(U S^{2 n}\right)$ induced by $\widehat{\delta_{M}}$ (see Section 3.2). First, let us recall that

$$
\mathbb{H}_{*}\left(S^{2 n}\right) \cong \mathbb{Z}\left[k_{-2 n}\right] /\left(k_{-2 n}^{2}\right)
$$

and

$$
\mathbb{H}_{*}\left(U S^{2 n}\right) \cong \mathbb{Z}\left[g_{-4 n+1}, h_{-2 n}\right] /\left(g_{-4 n+1}^{2}, g_{-4 n+1} h_{-2 n}, 2 h_{-2 n}, h_{-2 n}^{2}\right) .
$$

Lemma 4.3 The $\mathbb{H}_{*}\left(S^{2 n}\right)$-module structure on $\mathbb{H}_{*}\left(U S^{2 n}\right)$ is given by the following products: $k_{-2 n} \cdot g_{-4 n+1}=0, k_{-2 n} \cdot h_{-2 n}=0$ and $k_{-2 n} \cdot 1_{U S^{2 n}}=h_{-2 n}$.

Proof The two first products vanish for degree reasons. For the last product, let us consider the canonical fibration $f: U S^{2 n} \rightarrow S^{2 n}$. By Theorem 2 of [14], the pullback diagram

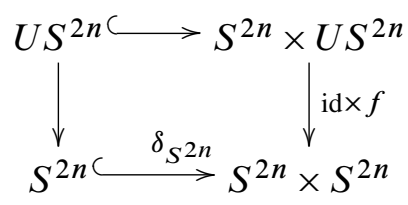




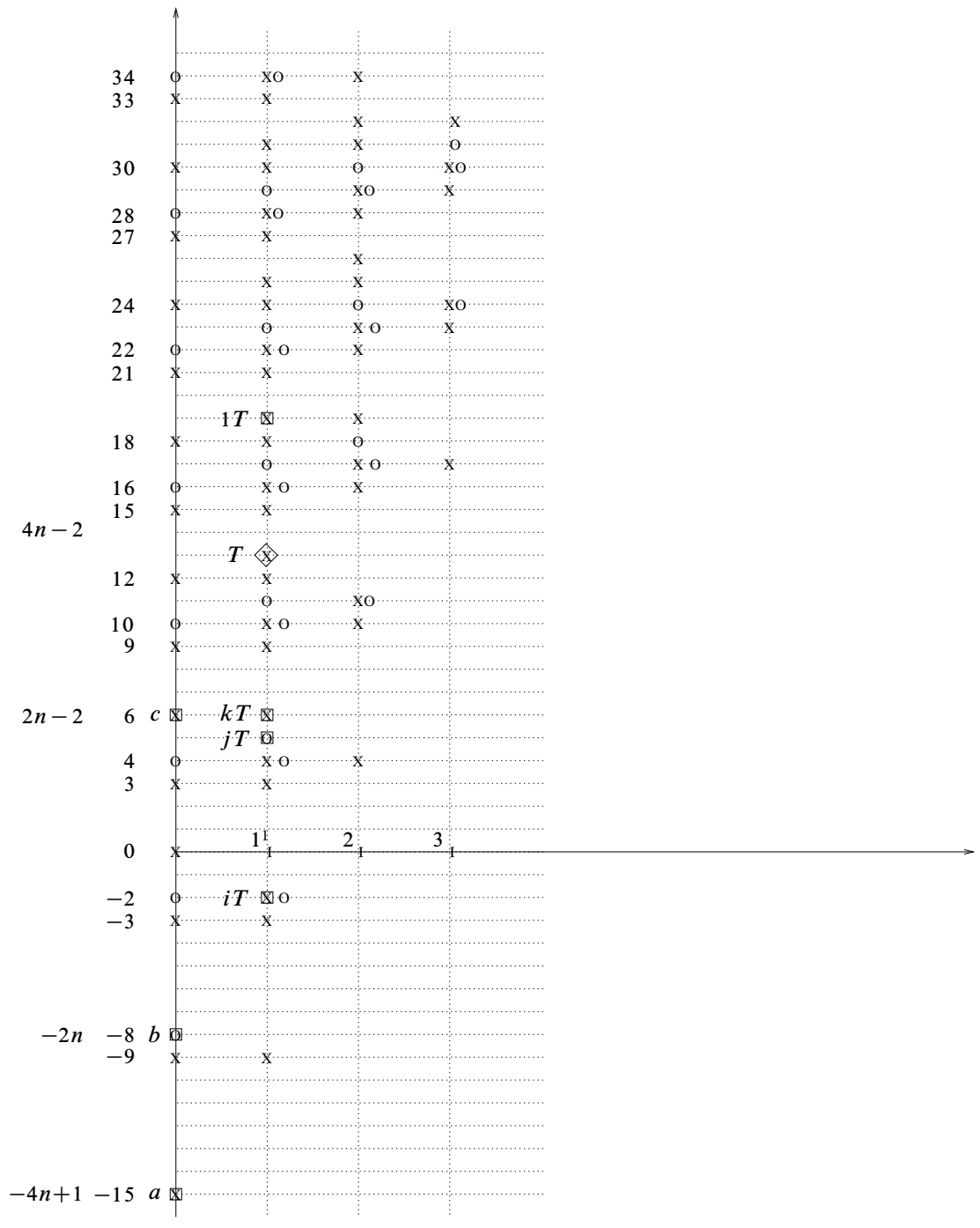

Figure 4

induces a structure of $\mathbb{H}_{*}\left(S^{2 n}\right)$-module on the shifted Serre spectral sequence of the fibration $f: U S^{2 n} \rightarrow S^{2 n}$. This structure is easy to compute. There is no extension issue for degree reasons. This proves Lemma 4.3.

Now, let us consider the two shifted multiplicative Serre spectral sequences associated to the fibrations $b: \widetilde{U S^{2 n}} \rightarrow U S^{2 n}$ and $c: \widetilde{S^{2 n}} \rightarrow S^{2 n}$. At the $E^{2}$-level, the module structure is given by the module structure of the preceding Lemma 4.3 on the base and by the Chas-Sullivan loop product on the fiber. Then, we have 
(1) $b . u_{2 n-2}=z_{-2 n} \cdot u_{2 n-2}$ and $b \cdot y_{-2 n+1}=z_{-2 n} y_{-2 n+1}$,

(2) $a . u_{2 n-2}=z_{-2 n} u_{2 n-2} y_{-2 n+1}$,

(3) $c . u_{2 n-2}=u_{2 n-2}^{2}, c . y_{-2 n+1}=u_{2 n-2} y_{-2 n+1}, c . z_{-2 n}=u_{2 n-2} z_{-2 n}$ and c. $x_{-4 n+1}=u_{2 n-2} x_{-4 n+1}$.

The other products vanish. For degree reasons, the only possible extension issue may be $c \cdot y_{-2 n+1} z_{-2 n}$. But since this product lifts to an element of $2-$ torsion, there is no extension issue.

\subsection{The multiplicative structure of $\mathbb{E}_{*, *}^{1}(\mathcal{M S})(\pi)$}

This subsection is the application of Theorem 2.9. Since we know all the columns of the Morse-Serre spectral sequence and its multiplicative structure, we have the following result.

Proposition 4.4 The algebra $\mathbb{E}_{*, *}^{1}(\mathcal{M S})(\pi)$ is isomorphic to

$$
\mathbb{Z}[a, b, c] /\left(b^{2}, 2 b, a^{2}, a b\right) \oplus \mathbb{Z}[i, j, k, l] /\left(i^{2}, 2 j, j^{2}, k^{2}\right)[T]
$$

with $\operatorname{deg}(a)=-4 n+1, \operatorname{deg}(b)=-2 n, \operatorname{deg}(c)=2 n-2$ and $\operatorname{deg}(i)=-4 n+1$, $\operatorname{deg}(j)=-2 n, \operatorname{deg}(k)=-2 n+1, \operatorname{deg}(l)=2 n-2, \operatorname{bideg}(T)=(1,4 n-3)$. The multiplication between an element of the $0-$ th column and an element of another column is given by $c . i=l . i, c . j=l . j, c . k=l . k, c . l=l^{2}, b . k=j . k, b . l=l . j$, $a . l=k \cdot l . j$.

This can be illustrated by the diagram in Figure 4. On this diagram, we have represented the generators $a, b, c$ of the 0 -th column and the generators $i, j, k, l$ of the first column by squares. We also put a diamond $\diamond$ around the generator $T$. We recall that this generator is of bidegree $(1,4 n-3)$ so that its total degree is $4 n-2=\alpha_{1}$.

\subsection{Comparison with the Cohen-Jones-Yan spectral sequence}

Let us determine the differentials of the Morse-Serre spectral sequence. Let us recall the $E^{\infty}$-page of the Cohen-Jones-Yan spectral sequence computed in [14], shown in Figure 5 .

The following proposition describes the multiplicative structure of this spectral sequence. 


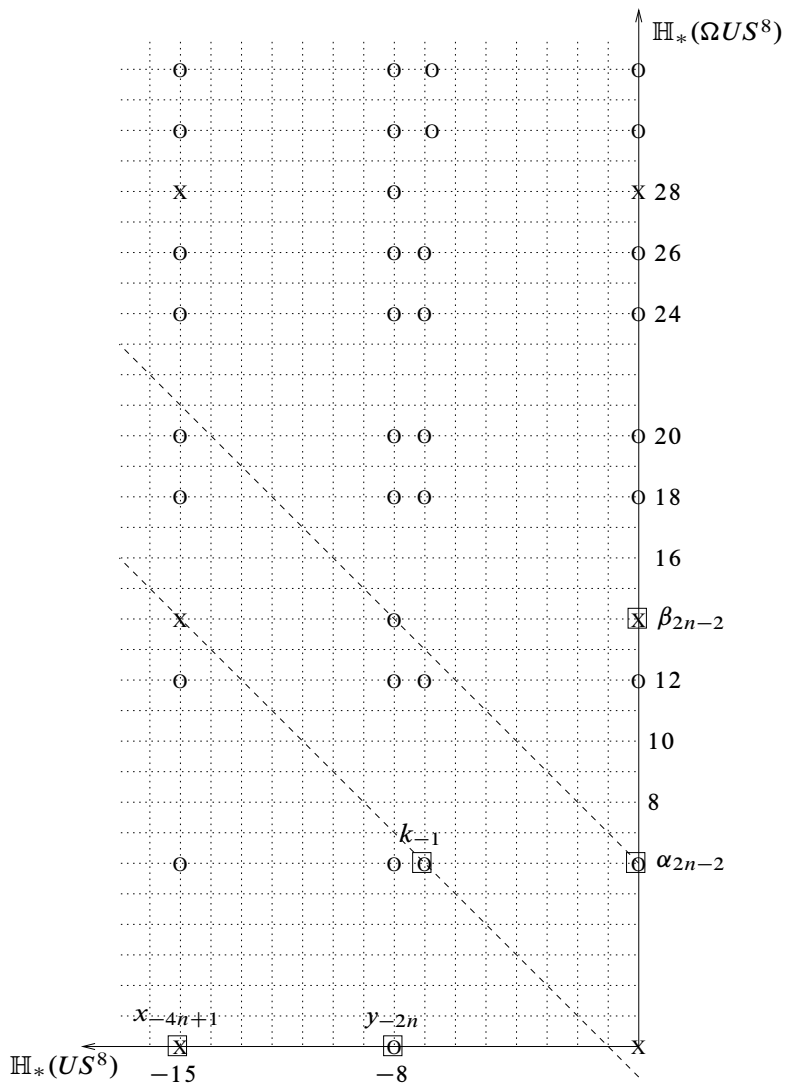

Figure 5

Proposition 4.5 The algebra $\mathbb{E}_{*, *}^{\infty}[\operatorname{ev}(0)]$ is isomorphic to

$$
\mathbb{Z}\left[x_{-4 n+1}, y_{-2 n}, \alpha_{2 n-2}, \beta_{4 n-2}, k_{-1}\right] / \mathcal{I}
$$

with $\mathcal{I}$ be the ideal generated by

$$
\begin{aligned}
&\left\{x_{-4 n+1}^{2}, x_{-4 n+1} y_{-2 n}, x_{-4 n+1} k_{-1}, 2 y_{-2 n}, y_{-2 n}^{2},\right. \\
&\left.y_{-2 n} k_{-1}-x_{-4 n+1} \alpha_{2 n-2}, 2 k_{-1}, 2 \alpha_{2 n-2}\right\} .
\end{aligned}
$$

Proof As the Cohen-Jones-Yan spectral sequence collapses at the $E^{2}$-term, we just have to give a description by generators and relations of the graded algebra $\mathbb{H}_{*}\left(U S^{2 n} ; H_{*}\left(\Omega U S^{2}\right)\right)$. 
First notice that this algebra is generated by the subalgebra $\mathbb{H}_{*}\left(U S^{2 n}\right) \otimes H_{*}\left(\Omega U S^{2 n}\right)$ and an element $k_{-1} \in \mathbb{H}_{-2 n+1}\left(U S^{2 n}, H_{2 n-2}\left(\Omega U S^{2 n}\right)\right)=\mathbb{Z} / 2 \mathbb{Z}$. We recall that

$$
\mathbb{H}_{*}\left(U S^{2 n}\right) \otimes H_{*}\left(\Omega U S^{2 n}\right) \cong \mathbb{Z}\left[x_{-4 n+1}, y_{-2 n}, \alpha_{2 n-2}, \beta_{4 n-2}\right] / \mathcal{J}
$$

where the ideal $\mathcal{J}$ is generated by

$$
\left\{x_{-4 n+1}^{2}, x_{-4 n+1} y_{-2 n}, 2 y_{-2 n}, y_{-2 n}^{2}, 2 \alpha_{2 n-2}\right\} \text {. }
$$

Thus we are left with the computations of the relations involving $k_{-1}$ and the subalgebra $\mathbb{H}_{*}\left(U S^{2 n}\right) \otimes H_{*}\left(\Omega U S^{2 n}\right)$. As $k_{-1}$ is of 2-torsion we compute the Cohen-Jones-Yan spectral sequence for the coefficient ring $\mathbb{Z} / 2 \mathbb{Z}$ that we denote by $\mathbb{E}_{*, *}^{r}[\operatorname{ev}(0) ; \mathbb{Z} / 2 \mathbb{Z}]$. This spectral sequence also collapses at the $E^{2}$-term and as we work over a field we have

$$
\mathbb{E}_{*, *}^{\infty}[\operatorname{ev}(0) ; \mathbb{Z} / 2 \mathbb{Z}]=\mathbb{H}_{*}\left(U S^{2 n} ; \mathbb{Z} / 2 \mathbb{Z}\right) \otimes H_{*}\left(\Omega U S^{2 n} ; \mathbb{Z} / 2 \mathbb{Z}\right) .
$$

The algebra morphism

$$
\mathbb{E}_{*, *}^{\infty}[\operatorname{ev}(0) ; \mathbb{Z}] \rightarrow \mathbb{E}_{*, *}^{\infty}[\operatorname{ev}(0) ; \mathbb{Z} / 2 \mathbb{Z}]
$$

allows us to prove that $k_{-1} y_{-2 n}=x_{-4 n+1} \alpha_{2 n-2}$ and that $k_{-1} \beta_{4 n-2}$ and $k_{-1} \alpha_{2 n-2}$ don't vanish.

Let us now explain how to compute the nonzero differentials on $\mathbb{E}_{*, *}^{1}(\mathcal{M S})(\pi)$. The module $\mathbb{E}_{-4 n+1,2 n-2}^{\infty}[\operatorname{ev}(0)]$ is $\mathbb{Z} / 2 \mathbb{Z}$. Then, we must have

$$
d_{1}(k i T)=2 a c .
$$

Since $d_{1}(i T)=0$ by comparison with the Cohen-Jones-Yan spectral sequence, we must have

$$
d_{1}(k T)=2 c .
$$

The multiplication by $c \in \mathbb{E}_{0,2 n-2}^{1}(\mathcal{M S})(\pi)$ gives the differentials on $c^{\kappa} k T=l^{\kappa} k T$ and on $c^{\kappa} k i T=l^{\kappa} k T, \kappa \in \mathbb{N}$. The comparison with the Cohen-Jones-Yan spectral sequence allows us to conclude that they are the only nonzero differentials from the first column of $\mathbb{E}_{*, *}^{*}(\mathcal{M S})(\pi)$. We deduce the other differentials by multiplying by $T$. See Figure 6.

The new generators are on the two first columns of $\mathbb{E}_{*, *}^{2}(\mathcal{M S})(\pi)$. For degree reasons there could not have nonzero differentials on this page and on the others. Thus we have $\mathbb{E}_{*, *}^{2}(\mathcal{M S})(\pi)=\mathbb{E}_{*, *}^{\infty}(\mathcal{M S})(\pi)$. See Figure 7 


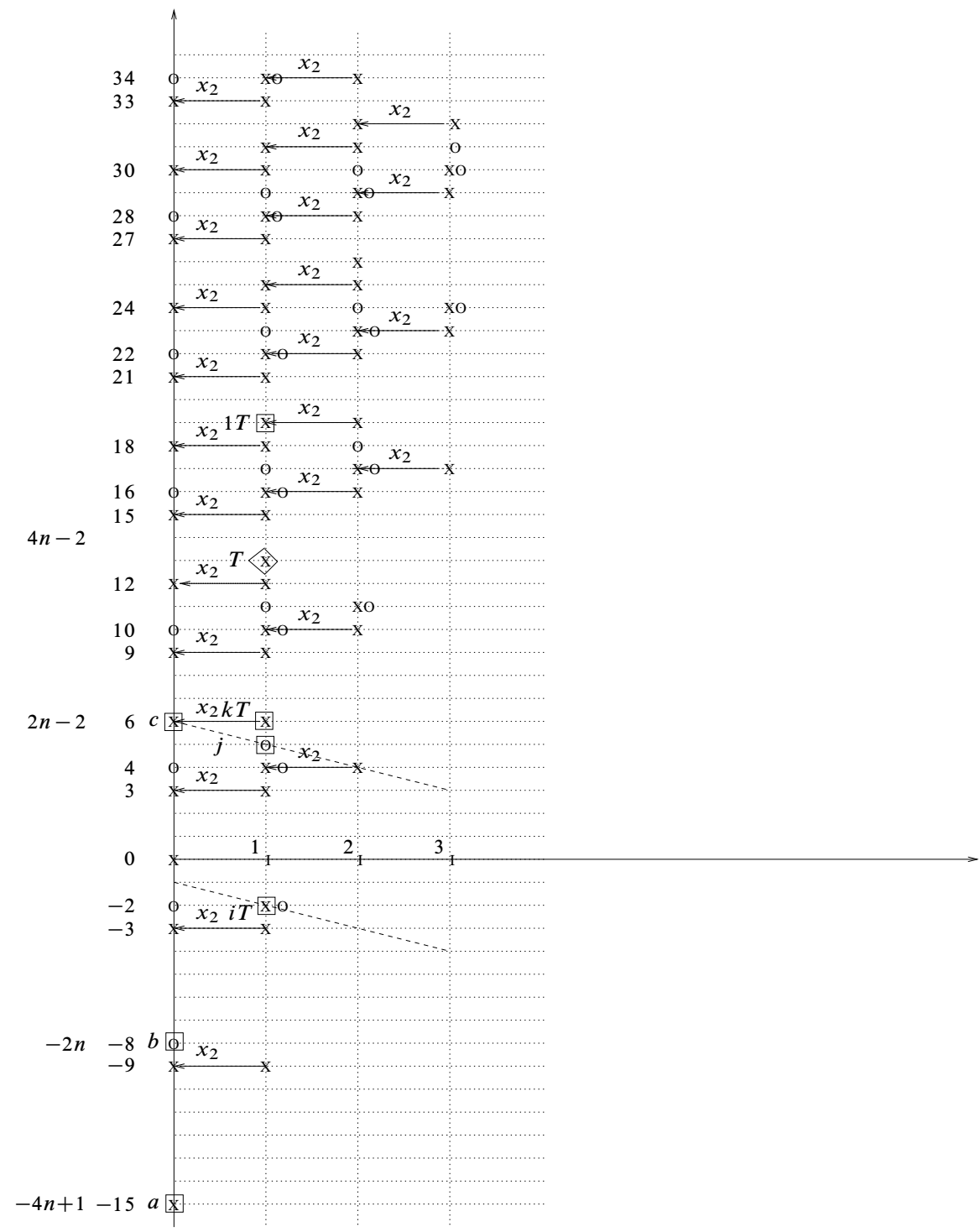

Figure 6

\subsection{Solve the linear extension issues}

Now that we know the $E^{\infty}$-terms of the two spectral sequences, we have to compare them to solve their extension issues. In the Cohen-Jones-Yan spectral sequence we have two linear extensions issues. They are located in total degree -1 and in degree $2 n-2$. 


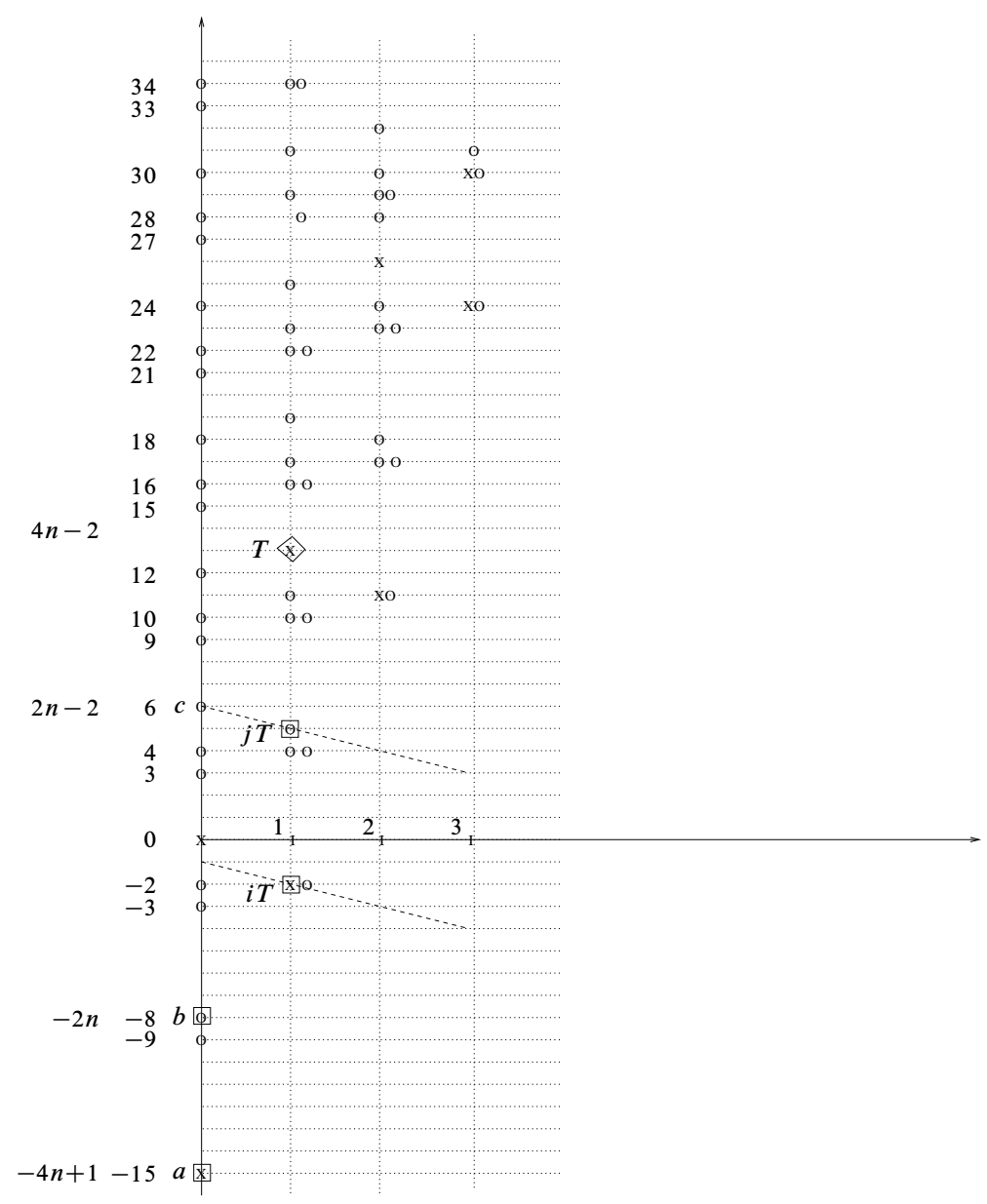

Figure 7

Proposition 4.6 The module $\mathbb{H}_{2 n-2}\left(\mathcal{L} U S^{2 n}\right)$ is equal to $\mathbb{Z}_{2} \oplus \mathbb{Z}_{2}$.

Proof To solve this extension issue, we use the multiplicative structure of the spectral sequences. By our preceding computations we have to decide if $\mathbb{H}_{2 n-2}\left(\mathcal{L} U S^{2 n}\right) \simeq$ $\mathbb{Z}_{2} \oplus \mathbb{Z}_{2}$ or $\mathbb{Z}_{4}$. Let $x_{2 n-2}$ be an element of $\mathbb{H}_{2 n-2}\left(\mathcal{L} U S^{2 n}\right)$ that represents the element $\alpha_{2 n-2}$ in the Cohen-Jones-Yan spectral sequence and let $x_{-4 n+1}$ be the element of lowest degree in $\mathbb{H}_{*}\left(\mathcal{L} U S^{2 n}\right)$. Then, the product $x_{2 n-2} x_{-4 n+1}$ lies in $\mathbb{E}_{0,-2 n-1}^{\infty}(\mathcal{M S})(\pi)$. This proves that $x_{2 n-2}$ lies in $F_{0} \mathbb{H}_{2 n-2}\left(\mathcal{L} U S^{2 n}\right)$ according to the Morse-Serre filtration and that this generator can not be of 4-torsion but of 2-torsion. 


\subsection{Solve the extension issues of algebra}

Proposition 4.7 There is no extension issues of algebra on the Cohen-Jones-Yan spectral sequence $\mathbb{E}_{*, *}^{\infty}[\mathrm{ev}(0)]$.

Proof Now that we have solve the linear extension issues, we can write that $x_{-4 n+1}$, $y_{-2 n}, \alpha_{2 n-2}, \beta_{4 n-2}$ and $k_{-1}$ as elements of $\mathbb{H}_{*}\left(\mathcal{L} U S^{2 n}\right)$.

(1) From the Morse-Serre filtration, we know that $x_{-4 n+1}, y_{-2 n}$ and $\alpha_{2 n-2}$ lie in $\mathbb{E}_{0, *}^{\infty}(\mathcal{M S})(\pi)$. Then, there is no extension issue of algebra on products between them.

(2) $x_{-4 n+1} k_{-1}=0$ for degree reasons.

(3) There is no extension issues concerning the products $x_{-4 n+1} \beta_{4 n-2}, y_{-2 n} k_{-1}$, $y_{-2 n} \beta_{4 n-2}$ and $k_{-1}^{2}$ because of there position in the filtration.

(4) On the Cohen-Jones-Yan spectral sequence, we see that there is a free module in the filtration preceding the product $\beta_{4 n-2} k_{-1}$. Since there is no 2 -torsion element in this module, there is no ambiguity.

(5) The possible ambiguity concerning $\beta_{4 n-2}^{2}$ is solved by computing $\mathbb{H}_{*}\left(\mathcal{L} U S^{2 n}, \mathbb{Q}\right)$. We use the fact that over the rational numbers $U S^{2 n}$ is homotopy equivalent to $S^{4 n-1}$ (the loop product is a rational homotopy invariant).

(6) The two last extension issues of algebra namely $\alpha_{2 n-2} k_{-1}$ and $\alpha_{2 n-2} \beta_{4 n-2}$ are solved by using the Morse-Serre spectral sequence where there are no ambiguities for these products.

\section{Computation of $\mathbb{H}_{*}\left(\mathcal{L} U S^{2 n+1}\right), n \geq 2$}

Strategy of the proof We compute $\mathbb{E}_{*, *}^{\infty}(\pi)$, the $E^{\infty}$ term of the $(2 n+1,2 n)-$ regraded Serre spectral sequence associated to the fibration $\pi: \mathcal{L} U S^{2 n+1} \rightarrow \mathcal{L} S^{2 n+1}$ in Section 5.1. There is no linear extension issue. Then, by computing the CohenJones-Yan spectral sequence $\mathbb{E}_{*, *}^{*}(\operatorname{ev}(0))$ in Section 5.2, and the Morse-Serre spectral sequence $\mathbb{E}_{*, *}^{*}(\mathcal{M S}(\pi))$ in Section 5.3, we solve the extension issues of algebra from $\mathbb{E}_{*, *}^{\infty}(\pi)$ in Proposition 5.4.

\subsection{The regraded Serre spectral sequence $\mathbb{E}_{*, *}^{*}(\pi)$}

We compute the last page of the $(2 n+1,2 n)$-regraded Serre spectral sequence of the fibration $\pi: \mathcal{L} U S^{2 n+1} \rightarrow \mathcal{L} S^{2 n+1}$. Then, we deduce the graded module structure of $H_{*}\left(\mathcal{L} U S^{2 n+1}\right)$. 
Proposition 5.1 The last page of $\mathbb{E}_{*, *}^{*}(\pi)$, namely $\mathbb{E}_{*, *}^{\infty}(\pi)$, is isomorphic to

$$
\mathbb{H}_{*}\left(\mathcal{L} S^{2 n+1}\right) \otimes \mathbb{H}_{*}\left(\mathcal{L} S^{2 n}\right)
$$

which is isomorphic to

$$
\mathbb{Z}\left[x_{-2 n-1}, v_{2 n}\right] /\left(x_{-2 n-1}^{2}\right)
$$

$$
\otimes \mathbb{Z}\left[y_{-2 n}, u_{4 n-2}, \theta_{-1}\right] /\left(y_{-2 n}^{2}, y_{-2 n} \theta_{-1}, \theta_{-1}^{2}, 2 y_{-2 n} u_{4 n-2}\right) .
$$

Proof Let us determine the $(2 n+1,2 n)$-regraded Serre spectral sequence of the fibration $\pi: \mathcal{L} U S^{2 n+1} \rightarrow \mathcal{L} S^{2 n+1}$. The Chas-Sullivan loop product provides this spectral sequence with a multiplicative structure (see Laudenbach [14]). Since $\mathbb{H}_{*}\left(\mathcal{L} S^{2 n+1}\right)$ has no torsion, $\mathbb{E}_{*, *}^{2}(\pi) \cong \mathbb{H}_{*}\left(\mathcal{L} S^{2 n+1}\right) \otimes \mathbb{H}_{*}\left(\mathcal{L} S^{2 n}\right)$. There are five generators of algebra: $x$ of degree $-(2 n+1), y$ of degree $-2 n, u$ of degree $4 n-2, v$ of degree $2 n$ and $\theta$ of degree -1 . This generators are represented by squares in Figure 8 .

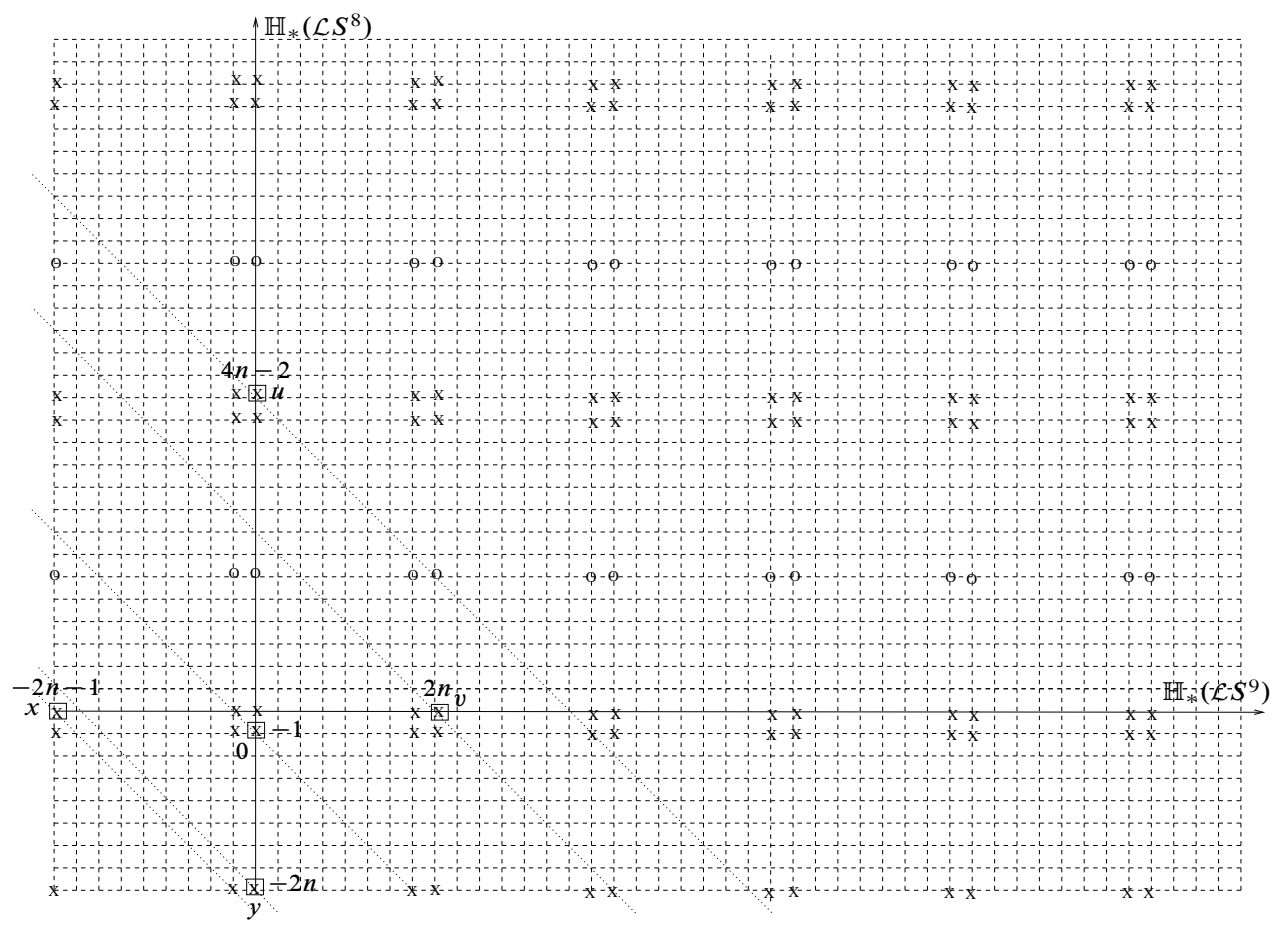

Figure 8

For degree reasons the only nonzero differential starting from a generator is $d_{2 n}$. More precisely, we only have to compute $d_{2 n}(y)$. Since there is a section $U S^{2 n+1} \rightarrow$ 
$\mathcal{L} U S^{2 n+1}$ and since that $\mathbb{H}_{*}\left(U S^{2 n+1}\right)=\mathbb{H}_{*}\left(S^{2 n+1}\right) \otimes \mathbb{H}_{*}\left(S^{2 n}\right)$, we have that $d_{2 n}(y)=0$. This proves that this spectral sequence collapse at the $E^{2}$-level and that the following figure represents $\mathbb{E}_{*, *}^{\infty}(\pi)$.

By an easy check we have the following proposition.

Proposition 5.2 There is no linear extension issue in $\mathbb{E}_{*, *}^{\infty}(\pi)$. Then, $H_{*}\left(L U S^{2 n+1}\right)$ is isomorphic to $H_{*}\left(\mathcal{L} S^{2 n+1}\right) \otimes H_{*}\left(\mathcal{L} S^{2 n}\right)$ as a graded module.

\subsection{The Cohen-Jones-Yan spectral sequence, $\mathbb{E}_{*, *}^{*}(\operatorname{ev}(0))$}

Since we know the module structure of $H_{*}\left(\mathcal{L} U S^{2 n+1}\right)$, we can deduce all the differentials of the Serre spectral sequence $\mathbb{E}_{*, *}^{*}(\operatorname{ev}(0))$ of the fibration ev(0): $\mathcal{L} U S^{2 n+1} \rightarrow$ $U S^{2 n+1}$.

Proposition 5.3 The algebra $\mathbb{E}_{*, *}^{\infty}(\mathrm{ev}(0))$ is isomorphic to

$$
\mathbb{Z}\left[a_{-2 n-1}, b_{-2 n}, c_{-1}, d_{2 n}, e_{4 n-2}\right] /\left(a_{-2 n-1}^{2}, b_{-2 n}^{2}, c_{-1}^{2}, c_{-1} b_{-2 n}, 2 e_{4 n-2} b_{-2 n}\right) .
$$

Proof Recall that $H_{*}\left(\Omega U S^{2 n+1}\right) \cong H_{*}\left(\Omega S^{2 n+1}\right) \otimes H_{*}\left(\Omega S^{2 n}\right)$. As $H_{*}\left(\Omega U S^{2 n+1}\right)$ is without torsion,

$$
\begin{aligned}
\mathbb{E}_{*, *}^{2}(\operatorname{ev}(0)) & \cong \mathbb{H}_{*}\left(U S^{2 n+1}\right) \otimes H_{*}\left(\Omega U S^{2 n+1}\right) \\
& \cong \mathbb{Z}\left[a_{-2 n-1}, b_{-2 n}, d_{2 n}, f_{2 n-1}\right] /\left(a_{-2 n-1}^{2}, b_{-2 n}^{2}\right)
\end{aligned}
$$

These generators are represented by squares on the diagram in Figure 9.

Since we know the linear structure of $\mathbb{H}_{*}\left(L U S^{2 n+1}\right)$, we can deduce all the differentials and compute $\mathbb{E}_{*, *}^{\infty}(\mathrm{ev}(0))$ together with its algebra structure.

\subsection{The Morse-Serre spectral sequence $\mathbb{E}_{*, *}^{*}(\mathcal{M S})(\pi)$}

As for the even case, we determine $\mathbb{E}_{1, *}^{1}(\mathcal{M S})(\pi)$ by computing the $(4 n+1,2 n)$ regraded Serre spectral sequence of the fibration

$$
b: \widetilde{U S^{2 n+1}} \rightarrow U S^{2 n+1} \text {. }
$$

Proposition 5.4 The subalgebra $\mathbb{E}_{p, *}^{1}(\mathcal{M S})(\pi), p \geq 1$, is isomorphic to

$$
\mathbb{Z}[\tau, v, \phi, \chi, \psi] /\left(\tau^{2}, v^{2}, \chi^{2}, \phi^{2}, 2 \psi \phi\right)[T]_{\geq 1}
$$

with $\operatorname{deg}(\tau)=-2 n-1, \operatorname{deg}(v)=-2 n, \operatorname{deg}(\phi)=-2 n, \operatorname{deg}(\chi)=-1, \operatorname{deg}(\psi)=4 n-2$ and $\operatorname{bideg}(T)=(1,4 n-1)$. 

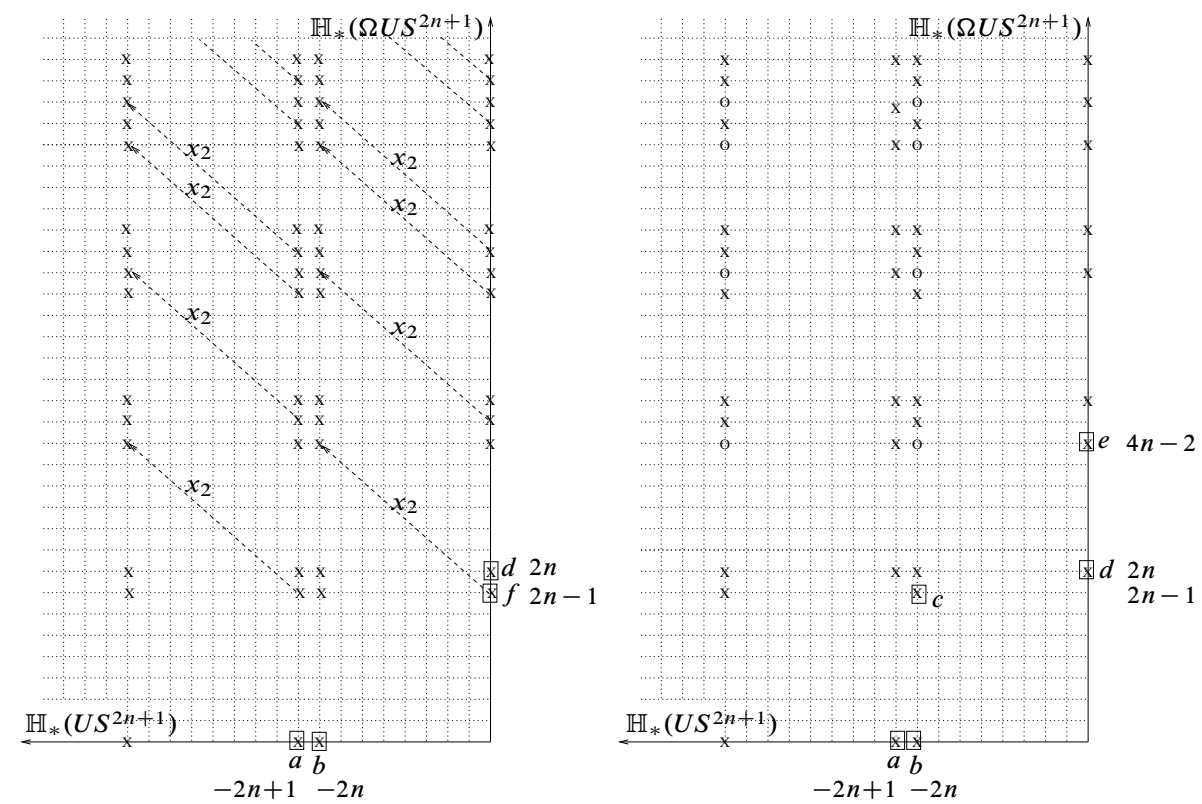

Figure 9

Proof We compute the $E^{2}$ term of the $(4 n+1,2 n)$-shifted Serre spectral sequence associated to the fibration $b: \widetilde{U S^{2 n+1}} \rightarrow U S^{2 n+1}$. See Figure 10 .

An easy inspection and the use of the section of $\pi: \widetilde{\Sigma_{1}} \rightarrow \Sigma_{1}$ as for the even dimensional case (Section 4.1), shows that all the differentials of this spectral sequence must vanish.

We deduce that $\mathbb{E}_{*, *}^{*}(\mathcal{M S})(\pi)$ collapses at the $E^{1}$ level and we get $\mathbb{E}_{*, *}^{\infty}(\mathcal{M S})(\pi)$. See Figure 11.

Proposition 5.5 The algebras $\mathbb{H}_{*}\left(\mathcal{L} U S^{2 n+1}\right)$ and $\mathbb{E}_{*, *}^{\infty}(\pi)$ are isomorphic.

Proof There may have ambiguities on the products of generators on $\mathbb{E}_{*, *}^{\infty}(\pi)$ for the following products.

(1) The products involving $\theta_{-1}, y_{-2 n}$ and $u_{4 n-2}$. These generators lie in the column $\mathbb{E}_{0, *}^{\infty}(\mathcal{M S})(\pi)$ so there is no multiplicative extension issue.

(2) The product $v_{2 n} \theta_{-1}$. We can drop the extension issue by considering the CohenJones-Yan spectral sequence $\mathbb{E}_{*, *}^{*}(\operatorname{ev}(0))$.

(3) The last extension issue concerning the product $v_{2 n} \theta_{-1}$ can be solved by considering the multiplicative structure of the Morse-Serre spectral sequence and to be more precise the $\mathbb{E}_{0, *}^{\infty}(\pi)$-module structure on $\mathbb{E}_{1, *}^{\infty}(\pi)$. 


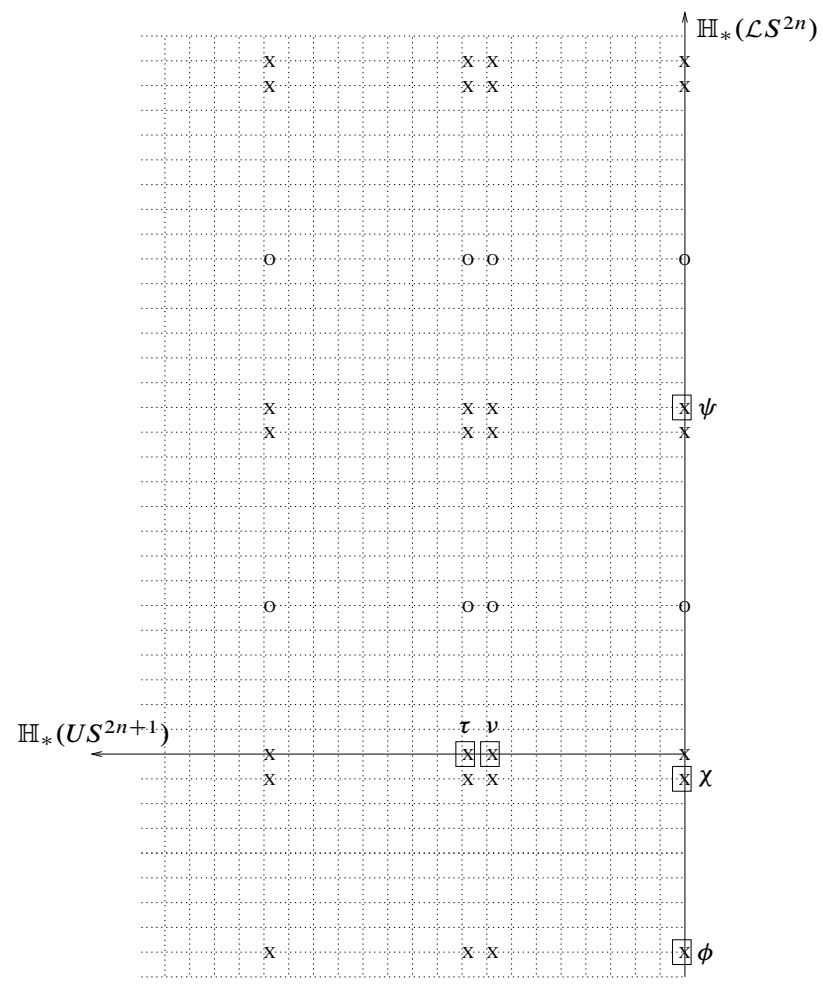

Figure 10

\section{References}

[1] A Abbondandolo, M Schwarz, Floer homology of cotangent bundles and the loop product, Preprint 41/2008, Max Planck Institute (2008)

[2] S Bauer, M Crabb, Polynomial loops on spheres, Q. J. Math. 55 (2004) 391-409 MR2104680

[3] R Bott, Nondegenerate critical manifolds, Ann. of Math. (2) 60 (1954) 248-261 MR0064399

[4] R Bott, On manifolds all of whose geodesics are closed, Ann. of Math. (2) 60 (1954) 375-382 MR0073993

[5] R Bott, Lectures on Morse theory, old and new, Bull. Amer. Math. Soc. (N.S.) 7 (1982) 331-358 MR663786

[6] A S Cattaneo, P Cotta-Ramusino, R Longoni, Configuration spaces and Vassiliev classes in any dimension, Algebr. Geom. Topol. 2 (2002) 949-1000 MR1936977

[7] M Chas, D Sullivan, String topology arXiv:math.GT/9911159 


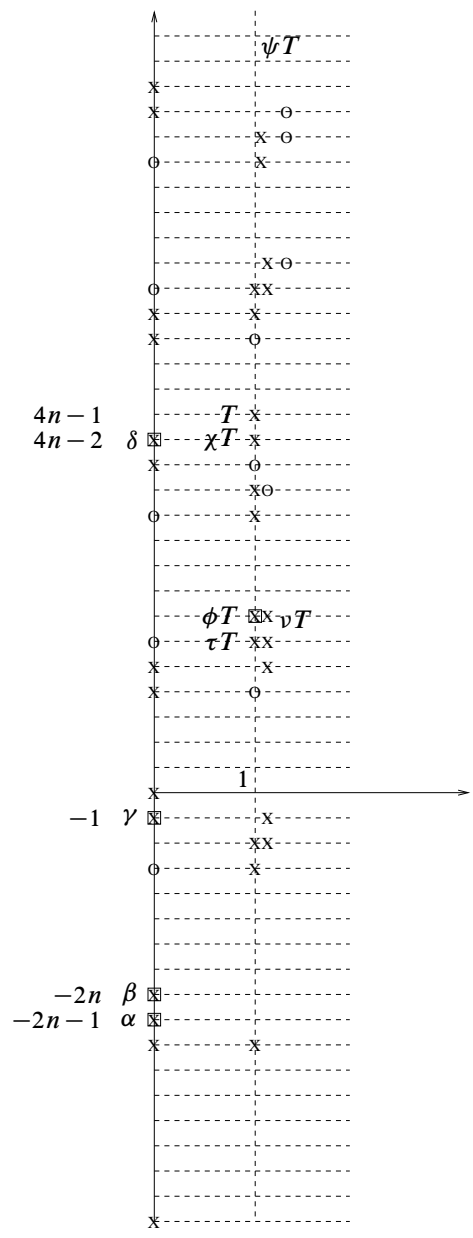

Figure 11

[8] E R Fadell, S Y Husseini, Geometry and topology of configuration spaces, Springer Monogr. in Math., Springer, Berlin (2001) MR1802644

[9] K Gruher, P Salvatore, Generalized string topology operations, Proc. Lond. Math. Soc. (3) 96 (2008) 78-106 MR2392316

[10] N Hingston, Loop products and closed geodesics arXiv:0707.3486

[11] M W Hirsch, Immersions of manifolds, Trans. Amer. Math. Soc. 93 (1959) 242-276 MR0119214

[12] M Hutchings, Floer homology of families. I, Algebr. Geom. Topol. 8 (2008) 435-492 MR2443235

[13] F Laudenbach, A note on the Chas-Sullivan product arXiv:0903.2801 
[14] J-F Le Borgne, The loop-product spectral sequence, Expo. Math. 26 (2008) 25-40 MR2384273

[15] R S Palais, Local triviality of the restriction map for embeddings, Comment. Math. Helv. 34 (1960) 305-312 MR0123338

[16] R S Palais, Lectures on the differential topology of infinite dimensional manifolds, Mimeographed notes by S Greenfield, Brandeis University (1964-1965)

[17] S Smale, Regular curves on Riemannian manifolds, Trans. Amer. Math. Soc. 87 (1958) 492-512 MR0094807

[18] S Smale, The classification of immersions of spheres in Euclidean spaces, Ann. of Math. (2) 69 (1959) 327-344 MR0105117

[19] D Sullivan, String topology background and present state, from: "Current developments in mathematics, 2005”, Int. Press, Somerville, MA (2007) 41-88 MR2459297

[20] W Ziller, The free loop space of globally symmetric spaces, Invent. Math. 41 (1977) 1-22 MR0649625

Laboratoire Paul Painlevé, Département de Mathématiques, Université de Lille1 59655 Villeneuve d'Ascq, France

David.Chataur@math.univ-lille1.fr, Leborgne. Jean-Francois@math.univ-lille1.fr

Received: 25 November $2008 \quad$ Revised: 26 March 2009 